\title{
Interchange Fee Rate, Merchant Discount Rate, and Retail Price in a Credit Card Network: A Game-Theoretic Analysis ${ }^{1}$
}

\author{
Hangfei GUO ${ }^{2}$, Mingming $\mathrm{LENG}^{3}$, Yulan WANG
}

APRIL 2011

Revised November 2011 and June 12, 2012

\footnotetext{
${ }^{1}$ The authors are grateful to the associate editor and two anonymous referees for their insightful comments that helped improve the paper.

${ }^{2}$ DeGroote School of Business, McMaster University, Hamilton, Ontario L8S 4M4, Canada. $<$ guoh24@mcmaster.ca $>$

${ }^{3}$ Corresponding author: Department of Computing and Decision Sciences, Lingnan University, Tuen Mun, Hong Kong. Research supported by the General Research Fund of the Hong Kong Research Grants Council under Research Project No. LU340409. <mmleng@ln.edu.hk>

${ }^{4}$ Institute of Textiles \& Clothing, The Hong Kong Polytechnic University, Hong Kong. $<$ Yulan.Wang@inet.polyu.edu.hk>
} 


\begin{abstract}
We consider two game-theoretic settings to determine the optimal values of an issuer's interchange fee rate, an acquirer's merchant discount rate, and a merchant's retail price in a credit card network. In the first setting, we investigate a two-stage game problem in which the issuer and the acquirer first negotiate the interchange fee rate, and the acquirer and the retailer then determine their merchant discount rate and retail price, respectively. In the second setting, motivated by the recent U.S. bill "H.R. 2695," we develop a three-player cooperative game in which the issuer, the acquirer, and the merchant form a grand coalition and bargain over the interchange fee rate and the merchant discount rate. Following the cooperative game, the retailer makes its retail pricing decision. We derive both the Shapley value- and the nucleolus-characterized, and globally-optimal unique rates for the grand coalition. Comparing the two game settings, we find that the participation of the merchant in the negotiation process can result in the reduction of both rates. Moreover, the stability of the grand coalition in the cooperative game setting may require that the merchant should delegate the credit card business only to the issuer and the acquirer with sufficiently low operation costs. We also show that the grand coalition is more likely to be stable and the U.S. bill "H.R. 2695" is thus more effective, if the degree of division of labour in the credit card network is higher as the merchant, the acquirer, and the issuer are more specialized in the retailing, the acquiring, and the issuing operations, respectively.
\end{abstract}

Key words: interchange fee rate; merchant discount rate; Nash bargaining; Stackelberg game; supermodularity; Shapley value; nucleolus. 


\section{Introduction}

In today's retailing market, many consumers consider the credit card payment as an important, dominating means to shop from merchants. As the Nilson Report indicates, 795.5 million MasterCard and Visa cards were held by the U.S. consumers in 2004 [13]. The consumers who intend to buy now and pay later are more likely to complete transactions with credit cards. In the United States, the aggregate credit card transaction amount was 1.7 trillion in 2003, and this number has been quickly growing [13]. For major benefits associated with credit-card transactions for both consumers and businesses, see, e.g., Hartman [18].

A typical credit card operation in reality usually involves two steps, as shown in Figure 1. In the first step, consumers buy products from the merchant and complete their transactions by confirming their credit card payments. In the second step, consumers pay the total credit-card transaction amounti.e., consumers' total expense that is calculated as the sum of the merchant's sales revenue (retail price times sales quantity) for all products - from their bank accounts to the issuer. Next, the issuer retains an interchange fee - that is computed as the transaction amount times an interchange fee rate $f$-as its revenue and transfers the remaining amount to the acquirer. The acquirer then charges the merchant a discount fee that equals the transaction amount times a merchant discount rate $d$. Note that the discount fee includes the issuer's interchange fee. That is, for an one-dollar credit card transaction, the acquirer obtains the merchant discount $d$ but pays the interchange fee $f$ to the issuer. It thus follows that the acquirer's revenue generated from this credit card business is $(d-f) \times$ total transaction amount. To assure the acquirer's non-negative profit, we realistically assume that $d \geq f$. As a result, the merchant's sales revenue is $(1-d) \times$ total transaction amount. For specific examples illustrating the credit card operation, see, e.g., Hunt [20].

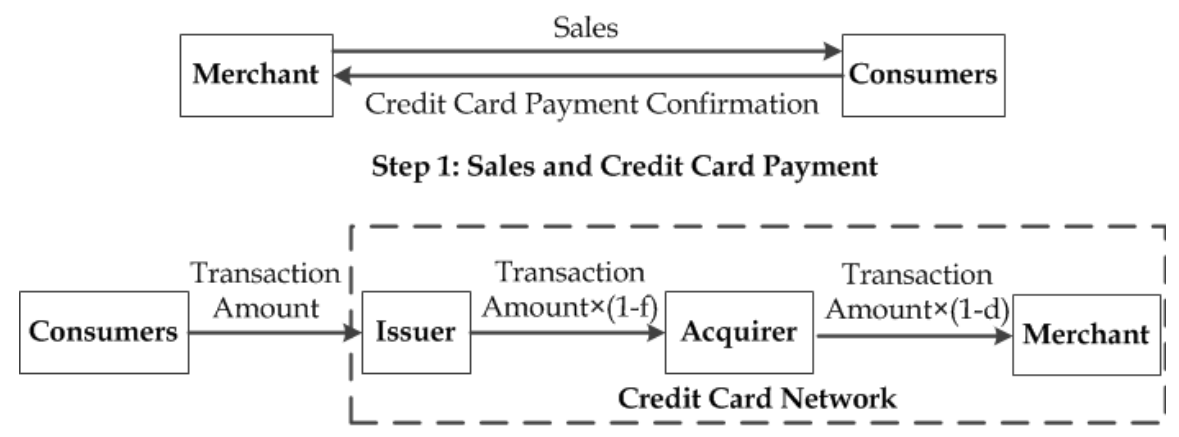

Step 2: Flow of Transaction Amount

Figure 1: The two-step credit card transactions. Note that $d$ and $f$ denote the merchant discount rate and the interchange fee rate, respectively.

In this paper, we consider a three-echelon credit card network that involves an issuer (a financial firm serving consumers), an acquirer (a financial firm serving the merchant), and a merchant serving consumers in a consumer market. The consumers use the credit cards (that are issued by the issuer) to buy from the merchant and pay their credit-card bills prior to the due dates. In the credit card network, the interchange fee is used to compensate the issuer for bearing the risk of issuing credit cards, and the merchant discount fee is used to motivate the acquirer to maintain the merchant's 
account and process credit card transactions. The interchange and merchant discount fees have been among the largest costs of merchants who accept the credit card payments (Akers et al. [1]). If the interchange fee rate is increased, then the issuer benefits more from the credit card operation, and the acquirer may accordingly raise its merchant discount rate so as to assure its profitability. As a result, the merchant's sales revenue may be reduced, and the merchant may respond by increasing its retail prices to improve its profit. However, a higher retail price may discourage consumers from buying the product, thereby resulting in a reduction in the total transaction amount and thus a decrease in the issuer's, the acquirer's, and the merchant's profits. Following the above facts, it is important to properly determine the interchange fee rate, the merchant discount rate, and the retail prices; but, such a decision problem has not been considered in existing publications, as indicated by our literature review in Section 2.

In practice, the interchange fee rate is either negotiated by the issuer and the acquirer or determined jointly by the issuer, the acquirer, and the merchant. Accordingly, we consider two game-theoretic settings to derive the optimal interchange fee rate, merchant discount rate, and retail price. In Sections 3 , we investigate the first setting that involves a two-stage game problem in which the merchant does not participate in the negotiation of the interchange fee rate. This scenario is consistent with the fact that the interchange fee rate is normally centrally determined by the credit card companies such as Visa and MasterCard, through a committee comprising at least partially representatives of issuers and acquirers (Small and Wright [39]). That is, in the first stage, the issuer and the acquirer bargain over the interchange fee rate, as discussed by Balto [3] and Small and Wright [39]. In the second stage, using the negotiated interchange fee rate, the acquirer determines a merchant discount rate and announces it to the merchant, who then makes its retail pricing decision. Note that our two-stage game involves a sequential financial decision problem in which, after an interchange fee rate is negotiated by the acquirer and the issuer, the acquirer determines a merchant discount rate, which is not negotiated by the acquirer and the merchant. The sequential decision problem is in gear with the fact that each acquirer has a real operation cost in addition to the interchange fee, and needs to create profit by adding a mark-up to the interchange fee, as discussed at MasterCard.com [27]. Since an acquirer's "profit" is actually a mark-up - i.e., the difference between the merchant discount and the interchange fee - minus the operation cost, we find that, given an interchange fee, the acquirer should make a decision on the markup or the merchant discount rate to "create" a profit. In fact, we can learn from MasterCard.com [27] that each major credit card network (e.g., Visa, MasterCard) announces its interchange fee rate publicly by, e.g., releasing the information online. This also implies that the interchange fee rate is negotiated before the merchant discount rate is determined by the acquirer. For extant publications concerning the above sequential financial decision problem, see, for example, Akers et al. [1] and Rochet and Tirole [33], who assumed that an acquirer's mark-up is its own operation (service) cost and set the merchant discount as the mark-up plus the interchange fee.

In the two-stage game setting, the second stage involves a sequential (Stackelberg) game in which the acquirer and the merchant act as the leader and the follower, respectively. We use backward induction to solve such a game. More specifically, in Section 3, we begin by solving the Stackelberg game between the acquirer and the merchant, assuming that the interchange fee rate is given. We then 
use the interchange fee rate-dependent Stackelberg solution to develop the issuer's and the acquirer's profit functions, and use the solution concept of Nash bargaining scheme [29] to characterize the negotiated interchange fee rate. We show that, in the two-stage game setting, the interchange-fee negotiation between the issuer and the acquirer helps entice the acquirer to stay in the credit card network.

In Section 4, we consider the second game setting that involves a three-player cooperative game in which the issuer, the acquirer, and the merchant bargain over the interchange fee rate and the merchant discount rate. This game is motivated by a recent U.S. legislation. In June 2009, John Conyers and Bill Shuster - who are the House Judiciary Committee Chairman Representatives - introduced the "Credit Card Fair Fee Act of 2009 (H.R. 2695)" [19] to the Senate and House of Representatives of the United States of America, which then enacted the bill to enable merchants to engage in collectively bargaining on a large scale with banks belonging to credit card networks such as Visa, MasterCard, etc. As John Conyers and Bill Shuster explained, this legislation would allow merchants to collectively negotiate with banks (i.e., acquirers and issuers) for certain credit card fees (i.e., interchange fee rate and merchant discount rate), and ultimately reduce the costs (i.e., retail prices) of everyday goods for consumers [50]. We note that, before 2009, many credit card companies (networks) did not allow merchants to directly bargain over the interchange fee rate with issuers and acquirers. But certain large merchants such as Wal-Mart could still bargain for a lower interchange fee rate (Akers et al. [1]).

We thus develop a two-step approach to find the rates for the cooperative game setting. Following the approach, we first construct a three-player cooperative game in characteristic-function form (von Neumann and Morgenstern [45, Ch. VI]). Then, we investigate whether there exist an interchange fee rate and a merchant discount rate so that the issuer, the acquirer, and the merchant are willing to cooperate for the credit card business. For similar approaches, see, e.g., Petrosjan and Zaccour [30], Sexton [36], etc. We show that, our three-player cooperative game is supermodular only when both the issuer and the acquirer can significantly contribute -i.e., generate sufficient profit surplusto the credit card network. We then apply Shapley value, the nucleolus, and the globally-optimal solution to determine the interchange fee rate and the merchant discount rate that result in a fair allocation of the system-wide profit among the three players. We find that, for most cases, the Shapley value-characterized rates cannot assure the non-empty core; but, we can always find the nucleoluscharacterized and the globally-optimal rates that guarantee the non-emptiness of the core and thus assure the stability of the grand coalition. Our cooperative game analysis for such a finance problem is an important focus of this paper; for other applications of the cooperative game theory in the financeoperation interface area, see, e.g., Gow and Thomas [17], where the concepts of Shapley value and the nucleolus were used to determine interchange fees for bank ATM networks.

Comparing our analytic results in the two game settings, we find that the participation of the merchant in the negotiation process indeed helps reduce both rates, as expected by the U.S. bill "H.R. 2695." We also show that the grand coalition is more likely to be stable and the U.S. bill "H.R. 2695" is thus more effective, if the degree of division of labour in the credit card network is higher as the merchant, the acquirer, and the issuer are more specialized in the retailing, the acquiring, and the issuing operations, respectively. This result may be justified by the fact that, in the United States, the 
degree of division of labour is high in the credit card business, as indicated by a recent report in [32].

Then, in Section 5, we discuss the implications for the two-player games with a single financial firm acting for both the acquiring and the issuing operations, and find that most results for the three-player games also hold for the two-player games. We also investigate the robustness of our results when we consider a logistic demand function, and find that our main managerial results hold for the game with the logistic function. Our paper ends with a summary of major concluding remarks in Section 6. The proofs of all theorems and a corollary are relegated to online Appendix A.

\section{Literature Review}

This paper is associated with those concerning retail pricing-, interchange fee rate-, and merchant discount rate-related problems in credit card networks. Most existing relevant papers have been reviewed by Chakravorti [11], Hunt [20], Bolt and Chakravorti [6], and Verdier [44]. Chakravorti [11] discussed the costs and benefits of credit cards to network participants, and summarized major publications that investigated certain key features in credit card networks, including merchant pricing policy, interchange fees, merchant acceptance, and network competition. Hunt [20] provided a brief overview of the economics of the payment card industry. Verdier [44] surveyed the recent literature about the interchange fee rate in payment card systems with an emphasis on the ongoing debate that opposes banks to the regulatory institutions or the competition authorities in various countries. Bolt and Chakravorti [6] classified relevant economic models for credit card operations into the following five categories: (i) models focusing on interchange fees; (ii) models with price differentiation at the point of sale; (iii) models with competition between networks; (iv) models accounting for the role of credit; and (v) models with competition among payment instruments. Our paper is closely related to those in categories (i) and (ii). Below we review some relevant works in these two categories.

Baxter [4] constructed a one-period model to investigate an interchange fee problem, assuming that the market is perfectly competitive for payment service and consumption goods. The author concluded that the interchange fee is an important and necessary tool that balances consumers' and the merchant's demands and the issuers' and the acquirers' costs. Schmalensee [34] extended Baxter's model in [4] by assuming that issuers and acquirers have significant market powers while merchants are perfectly competitive. Similar to Baxter [4], Schmalense [34] found that, as a balancing device, the interchange fee can increase the value of a credit card system by properly allocating costs between issuers and acquirers and thus reasonably determining the cost allocation between consumers and merchants. We note that both Baxter [4] and Schmalensee [34] ignored the strategic interactions of consumers and merchants.

As an early work involving the strategic interactions of consumers and merchants, Rochet and Tirole [33] constructed a quantitative model in which issuers have market powers but acquirers are perfectly competitive, and consumers and merchants decide rationally on whether to buy or to accept a payment card. They found that, due to the issuers' market powers, the socially-optimal interchange fee rate for the central planner is higher than that in Baxter [4]. Wright [48] assumed that the merchants may not accept credit cards for some strategic reasons, and developed a model in which the partial participation 
of heterogeneous consumers and merchants is allowed in a two-sided market. The author found that the privately-optimal interchange fee rate may be very high.

All of the above papers considered the centralized case in which the interchange fee rate is determined by a central planner or a credit card company/association such as Visa and MasterCard. However, Balto [3] questioned the rationality of the centrally-determined interchange fee rate, and argued that a lower interchange fee rate could be determined as a result of the bilateral negotiation between the acquirers and the issuers.

To the best of our knowledge, Small and Wright [39] is a seminal paper that assumed the interchange fee rate is negotiated rather than centrally determined. Small and Wright constructed a Nash bargaining model to characterize the bilateral bargaining over the interchange fee rate. They discussed the hold-up problem that results from the "honour all cards rule" and found that, as a result of implementing the rule, the bilaterally-negotiated interchange fee rate may be higher than the centrallydetermined interchange fee rate. However, Small and Wright [39] did not include the merchant's retail pricing decision in their model. In addition, Thomas [42] used the multi-player cooperative game theory to analyze the credit card-related problems. Motived by the major changes in the distribution of the credit card costs - e.g., the introduction of annual fees, the lowering of merchant service charges, etc.- in the U.K. at the end of 1980s, the author analyzed the fair allocation of the credit card-related costs among the credit card companies, the retailers who accept the credit card payments, and the cardholders. More specifically, Thomas [42] developed a credit-card cooperative game in the linear characteristic-value function, applied the concepts of the core, Shapley value, and the nucleolus to numerical examples, and performed a sensitivity analysis to examine the impacts of debit cards on credit-card fee structures. But, Thomas [42] did not analytically consider the interchange fee rate, merchant discount rate, and the retail prices.

Different from the aforementioned papers, we examine a two-stage game to investigate the negotiated interchange fee rate and to derive the optimal merchant discount rate and the optimal retail pricing decision. In addition, motivated by the recent U.S. legislation H.R. 2695 [19], we consider a three-player game where the acquirer, the issuer, and the merchant negotiate the interchange fee rate and the merchant discount rate. This significantly distinguishes our paper from the existing literature.

\section{The Two-Stage Game Analysis}

In this section, we assume that the acquirer and the issuer are two separate financial firms, which is in gear with the following fact: In a recent report released by Divisions of Research \& Statistics and Monetary Affairs in the U.S. Federal Reserve Board [32], Prager et al. listed the top 10 U.S. acquirers and the top 10 U.S. issuers, and their corresponding shares of all credit card transactions (in dollars) in 2007. From that report (see online Appendix B), we find that, in 2007, the top 10 acquirers and issuers held $88.7 \%$ and $89.6 \%$ of all acquiring- and issuing-related dollar value transactions, respectively. This shows that the top financial firms can properly represent the acquirers and issuers in the U.S. credit card business. In addition, among the top firms, only Bank of America is both a top acquirer with a share of $17.8 \%$ and a top issuer with a share of $14.6 \%$. Therefore, the percentage of the transactions by the 
firms acting mainly in the acquiring operation and by the firms acting mainly in the issuing operation can be roughly estimated as $(88.7 \%-17.8 \%) / 88.7 \%=79.9 \%$ and $(89.6 \%-14.6 \%) / 89.6 \%=83.7 \%$, respectively. The percentage of the transactions in the acquiring business and that in the issuing business by the firms acting in both operations are calculated as $20.1 \%$ and $16.3 \%$, respectively. The above percentages imply that the acquiring and the issuing operations are mostly performed by separate financial firms. Accordingly, in this paper, we focus on the three-player games involving a merchant, an acquirer, and an issuer, who are three separate firms. The implications for the two-player games with a merchant and a financial firm acting in both the acquiring and the issuing operations will be discussed later in Section 5.1.

Moreover, we learn from Section 1 that, in practice, the acquirer should first bargain with the issuer over the interchange fee rate, and secondly determine its merchant discount rate and announce it to the retailer, who then makes its retail price. Accordingly, we analyze a two-stage game to determine the interchange fee rate $f$, the merchant discount rate $d$, and retail price $p$ of a product in a credit card network.

1. In the first stage, the issuer and the acquirer bargain over the interchange fee rate, which can be modeled as a two-person cooperative game. We use the concept of Nash bargaining scheme to characterize the negotiated interchange fee rate. In this stage, the issuer and the acquirer (i) "cooperate" - jointly serve the credit card network - and (ii) "compete" - bargain over the interchange fee rate for the division of the profit resulting from the credit card operation - at the same time. This can be actually described as the "co-opetition," as discussed by Brandenburger and Nalebuff [8].

2. In the second stage, the acquirer determines and announces a merchant discount rate to the merchant, who then makes its retail pricing decision. This is a non-cooperative Stackelberg game in which the acquirer and the merchant act as the leader and the follower, respectively.

The above indicates that a cooperative game is followed by a non-cooperative game, which appears to be an interesting game structure. Such a structure differs from biform games discussed by Branden$\leftarrow$ EIC.BS burger and Stuart [7], who considered a two-stage game model involving a non-cooperative game in the first stage and a cooperative game in the second stage. Specifically, we learn from [7] that a biform game precisely reflects the idea that business strategies (in the non-cooperative setting) can shape the competitive environment in which players bargain over their payoffs. But, our two-stage game model indicates that some players' cooperative behaviors can determine a non-cooperative setting in which those and other players choose their business strategies.

One may note that, in reality, the merchant (e.g., the AEON Japan Group) may not cooperate with the external acquirer and issuer but instead operate by itself for the acquiring and the issuing business, which is actually unusual. In this section, we focus on the analysis of the two-stage game, which is a traditional and usual setting. Our analysis for the case with the merchant acting in both the acquiring and the issuing operations is provided in online Appendix C.1. Next, we use backward induction to solve this two-stage game problem. 


\subsection{The Analysis of the Stackelberg Game in the Second Stage}

Given the interchange fee rate $f$, we derive the merchant's best-response retail price $p^{*}(d, f)$, and then find the Stackelberg solution $\left(d^{*}(f), p^{*}(f)\right)$.

\subsubsection{The Merchant's Best-Response Pricing Decision}

The merchant buys the product at the unit acquisition cost $c$, and sells it at the retail price $p$. The demand for the product, denoted by $q(p)$, is a deterministic, linear, and price-dependent function, which is given as $q(p)=\alpha-\beta p$, where $\alpha>0$ denotes the price-independent demand and $\beta>0$ represents the marginal impact of the price. This assumption has been widely used in economics, finance, and operations management; see Bertrand [5], Corbett and Karmarkar [12], Lim and Ho [24], and the references therein.

To assure that the demand $q(p)$ is non-negative, we assume that the parameter $\alpha$ is sufficiently large such that $\alpha \geq \beta c$. Moreover, to generate the non-negative sales of the product, the merchant should determine the retail price such that $p \leq \alpha / \beta$. Thus, $(p-c) / p=1-c / p \leq(\alpha-\beta c) / \alpha$. Note that the ratio $(p-c) / p$ is regarded as the well-known "Lerner index" [23]. Lerner index reflects a firm's market power; that is, a firm with a higher value of Lerner index has a greater power in the market that it serves. Therefore, to assure that $q(p) \geq 0$, the merchant's market power for the product should be smaller than or equal to $(\alpha-\beta c) / \alpha$, which is the relative measure of the maximum demand for the product (i.e., $\alpha-\beta c$ ) over the constant, price-independent demand $\alpha$. We can compute the merchant's sales revenue and total acquisition cost as $R \equiv p \times q(p)=p(\alpha-\beta p)$ and $C \equiv c \times q(p)=c(\alpha-\beta p)$, respectively.

In practice, some merchants may accept both credit card payments and other common payment methods such as cash, checks, debit cards, etc., whereas other merchants may be unwilling to accept credit cards as a transaction medium. Since the early 1990s, among all payment methods, the percentage of the transaction dollar volume by credit cards has been rapidly increasing. We learn from Mann [25] that, as the U.S. Department of Commerce statistics indicates, credit card volume in 1999 accounted for about $23 \%$ of the value paid in all U.S. consumer payment transactions. The Association for Financial Professionals reported in [2] that, in 2005, 47\% of total transaction dollar volume at the U.S. retailers were based on consumer purchases with credit cards. For details regarding the U.S. purchase transactions (in dollars) with credit cards from 1990 to 2007, see the statistics released by Divisions of Research \& Statistics and Monetary Affairs in the U.S. Federal Reserve Board [32]. Based on the above facts, we assume that a percentage of the merchant's sales revenue $R$ is generated from the consumer purchases with the credit card. Note that, in reality, a consumer's willingness of using a credit card for his or her purchases is mainly affected by (i) the fixed fee (including, e.g., the annual fee) charged by the issuer - denoted by $F$-and (ii) the density of the issuer's network - denoted by $\eta \in[0,1]$, which reflects how widespread the issuer's network is. Accordingly, we assume that the percentage of consumptions with the credit card is dependent on $F$ and $\eta$. Hereafter, we denote such a percentage by $\tau(F, \eta)$, which should be decreasing in $F$ but increasing in $\eta$, i.e., $\partial \tau(F, \eta) / \partial F \leq 0$ and $\partial \tau(F, \eta) / \partial \eta \geq 0$. As a result, the remaining sales revenue $[1-\tau(F, \eta)] R$ results from the transactions with other payment methods. 
If the merchant does not accept the credit card, then it can only serve the consumers who does not pay with the credit card, and can thus achieve the profit $[1-\tau(F, \eta)](R-C)$. If the merchant decides to join the credit card network, then its sales profit can be calculated as $R-C$. Noting that the merchant needs to pay the acquirer the merchant discount fee $T=\tau(F, \eta) d R$, we find that the merchant's profit as $R-C-T$. Therefore, given the merchant discount rate $d$, the merchant's profit is based on whether or not it accepts the credit card; that is,

$$
\pi_{M}(p)= \begin{cases}\pi_{M}^{N C}(p) \equiv[1-\tau(F, \eta)](p-c)(\alpha-\beta p), & \text { if does not accept the credit card; } \\ \pi_{M}^{C}(p) \equiv\{[1-\tau(F, \eta) d] p-c\}(\alpha-\beta p), & \text { if accepts the credit card }\end{cases}
$$

where the superscripts " $N C$ " and " $C$ " represent "no credit card" and "credit card," respectively. We note that, if the merchant decides to accept the credit card, then, in order to assure that $[1-\tau(F, \eta) d] p-$ $c \geq 0$, the acquirer should determine its merchant discount rate such that $d \leq(\alpha-\beta c) /[\tau(F, \eta) \alpha]$.

We can calculate the optimal prices maximizing $\pi_{M}^{N C}(p)$ and $\pi_{M}^{C}(p)$ in $(1)$ as $p_{1} \equiv(\alpha / \beta+c) / 2$ and $p_{2}(d) \equiv\{\alpha / \beta+c /[1-\tau(F, \eta) d]\} / 2$, respectively. Since $0 \leq f \leq d \leq(\alpha-\beta c) /[\tau(F, \eta) \alpha]<1$ and $0 \leq \tau(F, \eta) \leq 1$, we find that $p_{1} \leq p_{2}(d)$, which implies that the acceptance of credit card transactions would induce the merchant to raise its retail price. Moreover, we find that, as $d$ increases when the merchant accepts the credit card, the merchant should raise its retail price. Substituting $p_{1}$ and $p_{2}(d)$ into $\pi_{M}^{N C}(p)$ and $\pi_{M}^{C}(p)$, respectively, we obtain the merchant's maximum profit without credit card transactions as $\pi_{M}^{N C}\left(p_{1}\right)=\beta[1-\tau(F, \eta)](\alpha / \beta-c)^{2} / 4$ - which is independent of $d$ - and that with credit card transactions as $\pi_{M}^{C}\left(p_{2}(d)\right)=\beta[1-\tau(F, \eta) d]\{\alpha / \beta-c /[1-\tau(F, \eta) d]\}^{2} / 4$ - which is decreasing in $d$ as $\partial\left[\pi_{M}^{C}\left(p_{2}(d)\right)\right] / \partial d<0$.

As $\lim _{d \rightarrow 0} \pi_{M}^{C}\left(p_{2}(d)\right)=\beta(\alpha / \beta-c)^{2} / 4 \geq \pi_{M}^{N C}\left(p_{1}\right)$ and $\lim _{d \rightarrow(\alpha-\beta c) /[\tau(F, \eta) \alpha]} \pi_{M}^{C}\left(p_{2}(d)\right)=0<$ $\pi_{M}^{N C}\left(p_{1}\right)$, there must exist a unique value $\kappa$ such that $\left.\pi_{M}^{C}\left(p_{2}(d)\right)\right|_{d=\kappa}=\pi_{M}^{N C}\left(p_{1}\right)$. Solving $\pi_{M}^{C}\left(p_{2}(d)\right)=$ $\pi_{M}^{N C}\left(p_{1}\right)$, we have,

$$
\kappa \equiv \frac{\alpha-\beta c}{\tau(F, \eta) \alpha}\left[1-\frac{\sqrt{[1-\tau(F, \eta)]^{2}(\alpha-\beta c)^{2}+4 \alpha \beta c[1-\tau(F, \eta)]}-[1-\tau(F, \eta)](\alpha-\beta c)}{2 \alpha}\right],
$$

which is greater than zero but smaller than $(\alpha-\beta c) /[\tau(F, \eta) \alpha]$. If $d \leq \kappa$, then $\pi_{M}^{C}\left(p_{2}(d)\right) \geq \pi_{M}^{N C}\left(p_{1}\right)$, and the merchant should accept the transactions with credit cards; otherwise, the merchant should not accept credit card transactions.

In order to induce the merchant to participate in the credit card network, the acquirer should set its merchant discount rate $d \leq \kappa$. As a result, when the merchant accepts the credit card, the $d$-dependent sales revenue is calculated as,

$$
R(d)=p_{2}(d) \times q\left(p_{2}(d)\right)=\frac{1}{4}\left[\frac{\alpha^{2}}{\beta}-\frac{\beta c^{2}}{[1-\tau(F, \eta) d]^{2}}\right] ;
$$

and the merchant's profit is computed as,

$$
\pi_{M}(d)=\pi_{M}^{C}(p)=\frac{1}{4} \frac{\{[1-\tau(F, \eta) d] \alpha-\beta c\}^{2}}{\beta[1-\tau(F, \eta) d]}
$$


which is decreasing in $d$ and $\tau(F, \eta)$; that is, the merchant's profit is reduced when the acquirer increases its merchant discount rate $d$ or more consumers prefer to shop with credit cards.

\subsubsection{The Interchange Fee Rate-Dependent Stackelberg Solution}

From Figure 1, we find that the acquirer's revenue can be obtained as $(d-f)$ times the total credit card transaction amount $\tau R(d)$, where $R(d)$ is given as in (3). Assume that the acquirer incurs an operation cost $c_{A}$ per dollar of credit-card transaction. Hence, given the interchange fee rate $f$, the acquirer determines its merchant discount rate by maximizing the following constrained problem,

$\max _{d} \pi_{A}(d)=\left(d-f-c_{A}\right) \tau(F, \eta) R(d)=\frac{\tau(F, \eta)\left(d-f-c_{A}\right)}{4}\left\{\frac{\alpha^{2}}{\beta}-\frac{\beta c^{2}}{[1-\tau(F, \eta) d]^{2}}\right\}$, s.t. $f+c_{A} \leq d \leq \kappa$,

where $d-f-c_{A}$ represents the acquirer's net profit per dollar of credit-card transaction; and the constraint (i.e., $\left.f+c_{A} \leq d \leq \kappa\right)$ guarantees the participation of both the acquirer and the merchant in the credit card business.

Theorem 1 The optimal interchange fee rate-dependent merchant discount rate $d^{*}(f)$ is

$$
d^{*}(f)= \begin{cases}d_{1}(f), & \text { if } f \leq \hat{f}, \\ \kappa(\text { constant }), & \text { if } \hat{f} \leq f \leq \kappa,\end{cases}
$$

where $d_{1}(f)$ is a unique solution satisfying the equation that $\alpha^{2}\left[1-\tau(F, \eta) d_{1}(f)\right]^{3}=\beta^{2} c^{2}\left[1+\tau(F, \eta) d_{1}(f)-\right.$ $\left.2 \tau(F, \eta)\left(f+c_{A}\right)\right] ;$ and,

$$
\hat{f} \equiv \frac{\left[1+\kappa \tau(F, \eta)-2 c_{A} \tau(F, \eta)\right] \beta^{2} c^{2}-[1-\kappa \tau(F, \eta)]^{3} \alpha^{2}}{2 \beta^{2} c^{2} \tau(F, \eta)}
$$

We also find that $d_{1}(f)$ is increasing in $f$, i.e., $\partial\left[d_{1}(f)\right] / \partial f \geq 0$; but, $\partial\left[d_{1}(f)\right] / \partial f<1$. Moreover, $d_{1}(f)$ is a convex function of $f$, i.e., $\partial^{2}\left[d_{1}(f)\right] / \partial f^{2} \geq 0$.

Substituting $d^{*}(f)$ (given in the above theorem) into $p_{2}(d)$ — which is given in Section 3.1.1-yields the $f$-dependent Stackelberg retail price for each product. In addition, Theorem 1 indicates that, if the interchange fee rate $f$ rises, then the acquirer should respond by increasing its merchant discount rate. But, this may discourage the merchant to accept customers' purchases with credit cards. In order to assure that the merchant is willing to trade with credit cards, the acquirer should bear a portion of the increase in the interchange fee rate. It thus follows that the increase in the merchant discount rate is smaller than the increase in the interchange fee rate.

\subsection{The Analysis of the Two-Player Cooperative Game in the First Stage}

We now investigate the first-stage game problem in which the acquirer and the issuer bargain over the interchange fee rate $f$, anticipating the $f$-dependent Stackelberg solution as obtained in Section 3.1. We next use the cooperative game concept of Nash bargaining scheme (NBS) [29] to characterize the negotiated interchange fee rate. The NBS represents a unique bargaining solution that can be obtained 
by solving the following maximization problem: $\max _{y_{1}, y_{2}}\left(y_{1}-y_{1}^{0}\right)\left(y_{2}-y_{2}^{0}\right)$, s.t. $y_{1} \geq y_{1}^{0}$ and $y_{2} \geq y_{2}^{0}$, where $y_{i}$ and $y_{i}^{0}$ correspond to player $i$ 's profit and security level (a.k.a. status quo point), respectively, for $i=1,2$. This concept has been broadly applied to analyze a variety of bargaining problems in the finance field; see, e.g., Cai [10], Ericsson and Renault [15], etc.

In our bargaining problem, we, w.l.o.g., assume that the acquirer and the issuer are player 1 and player 2, respectively. Thus, $y_{1}=\pi_{A}\left(d^{*}(f)\right)$, which can be easily obtained by substituting $d^{*}(f)$ in Theorem 1 into (5), and $y_{2}=\pi_{I}(f)$. Next, we compute the issuer's profit $\pi_{I}(f)$. The issuer can attain the interchange fee $f \times \tau \times R\left(d^{*}(f)\right.$ from the credit card transactions, where $R\left(d^{*}(f)\right)$ is found by replacing $d$ in $(3)$ with $d^{*}(f)$ in Theorem 1 . Moreover, as in practice, the issuer often charges each consumer who holds the credit card a fixed (transaction amount-independent) fee -including, e.g., the annual fee, the penalty for the late payment, and the expense over the credit limit - and a credit interest that is calculated as an interest rate times the consumer's transaction amount. For the fixed fees and the interest rates in recent U.S. credit card statistics, see Woolsey and Schulz [47]. Recalling from in Section 3.1.1 that the fixed fee is represented by $F$, we denote the interest rate for the credit card issued by the issuer by $t$. Therefore, the issuer's total revenue from the credit card transactions is calculated as $F+(f+t) \times \tau(F, \eta) \times R\left(d^{*}(f)\right.$. Letting $c_{I}\left(c_{I}<f\right)$ denote the issuer's operation cost per dollar of credit card transaction, we compute the issuer's profit as $\pi_{I}(f) \equiv F+\left(f+t-c_{I}\right) \times \tau(F, \eta) \times R\left(d^{*}(f)\right)$. For a similar calculation, see Brito and Hartley [9] who did not consider the fixed cost but only involved the interest rate into their model.

Note that neither the acquirer nor the issuer can gain any profit from credit-card transactions if they do not join the credit card network. Thus, the status quo point is $\left(y_{1}^{0}, y_{2}^{0}\right)=(0,0)$. Therefore, to derive the NBS-based interchange fee rate for the two-player cooperative game, we need to solve the maximization problem that $\max _{f} \Lambda(f) \equiv \pi_{A}\left(d^{*}(f)\right) \times \pi_{I}(f)$; that is,

$$
\max _{f} \Lambda(f)=\left\{\begin{array}{rlrl}
\Lambda_{1}(f) \equiv & \frac{\tau(F, \eta)\left[d_{1}(f)-f-c_{A}\right]}{4}\left[\frac{\alpha^{2}}{\beta}-\frac{\beta c^{2}}{\left[1-\tau(F, \eta) d_{1}(f)\right]^{2}}\right] \\
& \times\left\{F+\frac{\tau(F, \eta)\left(f+t-c_{I}\right)}{4}\left[\frac{\alpha^{2}}{\beta}-\frac{\beta c^{2}}{\left[1-\tau(F, \eta) d_{1}(f)\right]^{2}}\right]\right\}, \quad \text { if } c_{I} \leq f \leq \hat{f}, \\
\Lambda_{2}(f) \equiv & \left.\frac{\tau(F, \eta)\left[\kappa-f-c_{A}\right]\left[\frac{\alpha^{2}}{\beta}-\frac{\beta c^{2}}{4}\right]}{[1-\kappa \tau(F, \eta)]^{2}}\right] \\
& \times\left\{F+\frac{\tau(F, \eta)\left(f+t-c_{I}\right)}{4}\left[\frac{\alpha^{2}}{\beta}-\frac{\beta c^{2}}{[1-\kappa \tau(F, \eta)]^{2}}\right]\right\}, & & \text { if } \hat{f} \leq f \leq \kappa .
\end{array}\right.
$$

Next, we accordingly find the optimal interchange fee rate under the constraint $c_{I} \leq f \leq \hat{f}$ and that under the constraint $\hat{f} \leq f \leq \kappa$, which are then compared to obtain the NBS-characterized interchange fee rate.

Theorem 2 The functions $\Lambda_{1}(f)$ and $\Lambda_{2}(f)$ in $(7)$ are both log-concave in $f$. When the acquirer and the issuer bargain over the interchange fee rate, the NBS-based rate $f^{*}$ is uniquely determined as,

$$
f^{*}= \begin{cases}\tilde{f}_{2} \equiv \frac{\kappa+c_{I}-c_{A}-t-r_{1}}{2} \in[\hat{f}, \kappa], & \text { if } r_{1} \leq r_{2} \text { and } \Lambda_{1}\left(f_{1}^{*}\right) \leq \Lambda_{2}\left(\tilde{f}_{2}\right) \\ f_{1}^{*} \equiv \min \left(\tilde{f}_{1}, \hat{f}\right) \in\left[c_{I}, \hat{f}\right], & \text { otherwise }\end{cases}
$$


where

$$
r_{1} \equiv \frac{4 F}{\tau(F, \eta)\left\{\alpha^{2} / \beta-\beta c^{2} /[1-\kappa \tau(F, \eta)]^{2}\right\}} \quad \text { and } \quad r_{2} \equiv \frac{[1-\kappa \tau(F, \eta)]^{3} \alpha^{2}-\beta^{2} c^{2}}{\beta^{2} c^{2} \tau(F, \eta)}+c_{A}+c_{I}-t
$$

$\tilde{f}_{1}$ is the unique solution of the first-order condition that $\partial\left[\Lambda_{1}(f)\right] / \partial f=0$, and $\hat{f}$ is defined as in $(6)$.

As the above theorem implies, if the acquirer's and/or the issuer's per dollar operation costs (i.e., $c_{A}$ and $c_{I}$ ) are sufficiently small such that $r_{1} \geq r_{2}$, then the NBS-based interchange fee rate is no more than $\hat{f}$. Otherwise, the two players may choose a higher rate in the range $[\hat{f}, \kappa]$. This result happens simply because of the following reason: if the issuer incurs a high operation cost, then it should attempt to charge the acquirer a high interchange fee rate to offset its cost. As a response, the acquirer may increase its merchant discount rate. This may raise the acquirer's profit margin (i.e., the merchant discount rate minus the interchange fee rate) if the interchange fee rate is unchanged. However, by observing this, the issuer would accordingly bargain with the acquirer to increase its interchange fee rate.

In addition, Theorem 2 shows that, if the fixed fee $F$ or the interest rate $t$ is sufficiently large, then the value of $r_{1}$ is likely to be greater than the value of $r_{2}$, and the NBS-based interchange fee rate may be smaller than or equal to $\hat{f}$. That is, a large value of $F$ or $t$ may induce the issuer to bargain for a small interchange fee, because the issuer's high revenue from consumers can compensate for the reduction in the interchange fee. As Theorem 1 indicates, the acquirer responds by setting its merchant discount rate as $d_{1}(f)$, which is increasing in $f$.

Remark 1 Using $(3)$ and $d^{*}(f)$ in Theorem 1 to compute the issuer's profit $\pi_{I}(f)=F+(f+t-$ $\left.c_{I}\right) \times \tau(F, \eta) \times R\left(d^{*}(f)\right)$, we find that, when $\hat{f} \leq f \leq \kappa, \pi_{I}(f)$ is strictly increasing in $f$. That is, if the issuer determines the interchange fee rate by maximizing its own profit rather than by negotiating with the acquirer, then the optimal rate may be $f=\kappa$. Consequently, the acquirer may also set its merchant discount rate as $d=\kappa$. That is, if the interchange fee rate is determined by the issuer itself rather than by the negotiation, then the acquirer's profit would possibly be zero. This implies that the negotiation may help increase the acquirer's profit and entice the acquirer to stay in the credit card network.

\subsection{Stackelberg Equilibrium}

Substituting the NBS-characterized interchange fee rate $f^{*}$ into $\left(d^{*}(f), p_{2}\left(d^{*}(f)\right)\right)$, we can find the Stackelberg equilibrium $\left(d^{S}, p^{S}\right)$ as,

$$
\left(d^{S}, p^{S}\right)= \begin{cases}(\kappa,\{\alpha / \beta+c /[1-\tau(F, \eta) \kappa]\} / 2), & \text { if } r_{1} \leq r_{2} \text { and } \Lambda_{1}\left(f_{1}^{*}\right) \leq \Lambda_{2}\left(\tilde{f}_{2}\right) \\ \left(d^{*}\left(f_{1}^{*}\right), p_{2}\left(d^{*}\left(f_{1}^{*}\right)\right)\right), & \text { otherwise. }\end{cases}
$$

Next, we provide a numerical example to illustrate the NBS-characterized interchange fee rate, the merchant discount rate and retail price in Stackelberg equilibrium. 
Example 1 Suppose that the issuer's and the acquirer's operation costs per dollar of credit card transaction are $c_{I}=\$ 0.005$ and $c_{A}=\$ 0.004$, respectively. The parameters for the demand function are assumed to be $\alpha=100$ and $\beta=0.7$. In addition, the merchant's acquisition cost is $c=\$ 120$; the fixed fee and the interest rate for the credit card are $F=\$ 15$ and $t=0.002$ per dollar of credit card transaction. For all numerical examples in this paper, we specify the percentage of the merchant's sales revenue attributed to the credit card transactions as,

$$
\tau(F, \eta)=\rho_{1} \times \eta \times \exp \left(-\rho_{2} F\right)
$$

where $\rho_{i}>0$ (for $i=1,2$ ) are two parameters that are selected such that $\tau(F, \eta) \in[0,1]$. It is obvious that the above function $\tau(F, \eta)$ satisfies the property that $\partial \tau(F, \eta) / \partial F \leq 0$ and $\partial \tau(F, \eta) / \partial \eta \geq 0$. In this example, we assume that $\rho_{1}=10, \rho_{2}=0.15$, and $\eta=0.6$, which means that $\tau(F, \eta)=0.63$.

According to Theorem 2, we find that the NBS-characterized interchange fee rate is $f^{*}=4.31 \%$. Then, using (8), we obtain the Stackelberg merchant discount rate as $d^{S}=15.56 \%$, and the Stackelberg retail price of the product as $p^{S}=\$ 137.98$. $\triangleleft$

\section{The Cooperative Game Analysis}

In this section, we allow the merchant to negotiate with the acquirer and the issuer for the interchange fee rate and the merchant discount rate. This is different from Section 3 in which the merchant cannot bargain with other players over the rates. Correspondingly, we develop a three-player cooperative game to characterize the negotiation among the issuer, the acquirer, and the merchant, assuming that (i) players can communicate with each other and form coalitions with other players and (ii) players can make side payments to other players; see von Neumann and Morgenstern [45] and Straffin [40]. Note that the cooperative game theory with $n \geq 3$ players concerns the allocation of profit surplus or cost savings among the $n$ players who cooperate to form a grand coalition and achieve the surplus or savings.

In the credit card network, the issuer, the acquirer, and the merchant can join the three-player grand coalition and achieve a system-wide profit generated from the credit card business. To assure the stability of the grand coalition - i.e., the willingness of three players to cooperate for the credit card business, we should construct a three-player cooperative game (in the characteristic function form) and solve it for a fair allocation scheme. But, for our problem, we note that an allocation scheme among the three players is attributed to the interchange fee rate and the merchant discount rate in the grand coalition. For example, a higher value of the interchange fee rate results in more allocation to the issuer; similarly, increasing the merchant discount rate may raise the allocation to the acquirer. That is, given specific values of the two rates, we can compute the corresponding allocation scheme.

Next, we provide an approach to find the rates assuring that the grand coalition is stable; for similar approaches, see, e.g., Petrosjan and Zaccour [30], Sexton [36], etc. Specifically, in order to find the negotiated rates for the credit card network, we should consider the following two steps:

Step 1: Construct a cooperative game given the rates in the grand coalition. In this step, we construct a three-player cooperative game with the characteristic values in terms of the inter- 
change fee rate and the merchant discount rate in the grand coalition, which are assumed to be given.

Step 2: Search for the rates that assure the stability of the grand coalition. We solve the cooperative game to find a fair allocation scheme and the corresponding rates, assuring that the grand coalition is stable. We first derive the conditions for the non-empty core, in which each point represents a fair allocation scheme. In order to find a unique solution, we then compute Shapley value and the corresponding rates for the cooperative game. Note that (i) Shapley valuebased rates may not satisfy the conditions that assure the non-emptiness of the core, and (ii) Shapley value may not be in the core even if the core is non-empty. Thus, we need to examine whether or not Shapley value can be used to find the rates that result in the stability of the grand coalition. If Shapley value cannot assure that the grand coalition is stable, then we consider the nucleolus and the globally-optimal solution that maximizes the total profit in the grand coalition, which must exist in a non-empty core. For the nucleolus-based rates, we shall develop an algorithm to find the rates satisfying the conditions for the non-emptiness of the core.

Following the above two steps, we next develop a three-player cooperative game, and derive an interchange fee rate and a merchant discount rate that result in a fair allocation scheme assuring the stability of the grand coalition.

\subsection{The Three-Player Cooperative Game Given the Rates in the Grand Coalition}

To develop a cooperative game with three or more players, we need to compute the characteristic values of all possible coalitions. Note that the characteristic value of a coalition is the minimum profit that all members in the coalition can jointly achieve by using their own efforts only (von Neumann and Morgenstern [45]). For our three-player cooperative game, the possible coalitions are (i) the empty coalition $\{\varnothing\}$ in which no player joins the credit card network; (ii) three single-player coalitions $\{i\}$ $(i=M, A, I)$ in which only firm $i$ is involved in the credit card business; (iii) three two-player coalitions $\{i j\}(i, j=M, A, I, i \neq j)$, which represent the coalitions each involving firms $i$ and $j$ for the credit card business; and (iv) the three-player (grand) coalition $\{M A I\}$ in which all of three players cooperate to implement the credit card business.

Let $v(S)$ denote the characteristic value of the coalition $S$. Our three-player cooperative game in the characteristic-function form can be then written as: $v(\varnothing) ; v(M), v(A), v(I) ; v(M I), v(M A)$, $v(A I)$; and $v(M A I)$. Note that $v(\varnothing)=0$ since the profit generated by the empty coalition is naturally zero. We also find from the practice that the acquirer and the issuer, which are two banks, cannot participate in the retailing business. Therefore, if the merchant does not join the credit card network, then either the acquirer or the issuer or both of them cannot achieve any profit from the credit card business; that is, $v(A)=v(I)=v(A I)=0$. However, any coalition involving the merchant may gain a profit generated from consumer purchase with credit cards. The characteristic values $v(M), v(M I)$, $v(M A)$, and $v(M A I)$ are calculated as given in Table 1 , where $\delta \geq 1$ denotes the scale parameter for the acquiring-related operation cost of the merchant and the issuer in acting as an "acquirer;" and $\gamma \geq 1$ denotes the scale parameter for the issuing-related operation cost of the merchant and the 
acquirer in acting as an "issuer." Moreover, in Table $1, d^{S}$ is the Stackelberg equilibrium-characterized merchant discount rate in the two-stage game setting, as given in Section $3 ; f^{M I}$ and $d^{M A}$ denote the negotiated interchange fee rate and merchant discount rate in the coalitions $\{M I\}$ and $\{M A\}$, respectively. For details about our calculation, see online Appendix C.

For the three-player cooperative game, we need to clarify the role of the acquirer, which is a financial firm serving the merchant. This is important since, in practice, some large merchants (e.g., JUSCO, $\leftarrow$ AE.(2) Tesco, etc.) have their own banks, thus being bale to operate the acquiring business itself. That is, the merchant could act as an acquirer in addition to mainly participating in the retailing business. It then follows that we may not need a financial firm as the acquirer in the game, and the characteristic values $v(M I)$ and $v(M A I)$ may be identical. Actually, we can find from a number of practices that, in today's credit card networks, it is still common - and necessary - to have a separate financial firm as the acquirer, and $v(M I)$ and $v(M A I)$ are unlikely to be identical, because of the following two reasons.

1. In practice, if a large merchant possessing the financial function is willing to serve as an acquirer, then it, as usual, also decides to act as an issuer by issuing credit cards to consumers. This case corresponds to the coalition $\{M\}$, in which the merchant operates the credit card network by itself. Such an operation mainly stems from the merchant's motivations for the delivery of convenience to consumers and the improvement of profitability. The real examples include JUSCO and Tesco, as explained in online Appendix C.1.

2. In the retailing industry, most merchants - including a number of large merchants such as WalMart and Sears (which sold its retail credit card business to the Citibank in 2003) - do not participate in the financial operations, but focus on their retailing operations and cooperate with one or two external financial firms acting as the acquirer and the issuer. For example, Wal-Mart, GE Money Bank - which serves Wal-Mart as an acquirer, and the Citibank - which issues the Citibank credit cards to consumers who can use the cards to shop at Wal-Mart.

From the above, we conclude that (i) a separate financial firm as an acquirer widely exists in reality, because most merchants do not have their own banks, and (ii) most of the merchants with the financial functions usually undertake both the acquiring and the issuing operations rather than only the acquiring business.

We note from Table 1 that, in the coalition $\{M\}$ where the network density is $\eta_{M}$-which is smaller than $\eta$, the merchant's net operation cost for the credit card business $\delta c_{A}+\gamma c_{I}-t$ may not be less than the Stackelberg equilibrium merchant discount fee $d^{S}$, which is the "cost" of the merchant in the two-stage game setting. If $\delta c_{A}+\gamma c_{I}-t<d^{S}$, then, because $\tau\left(F, \eta_{M}\right)<\tau(F, \eta)$, the retail price $p^{S}$ and the merchant's profit $\pi_{M}\left(p^{S}\right)$ in the traditional setting are smaller than the retail price $p^{M_{1}}$ and the merchant's profit $\Pi_{M_{1}}\left(p^{M_{1}}\right)$ when the merchant acts alone in the credit card business, respectively. Otherwise, if $\delta c_{A}+\gamma c_{I}-t \geq d^{S}$, then $p^{S}$ and $\pi_{M}\left(p^{S}\right)$ may be greater than $p^{M_{1}}$ and $\Pi_{M_{1}}\left(p^{M_{1}}\right)$, respectively, which depends on the value of the fixed fee $F$ and the difference between $\tau\left(F, \eta_{M}\right)$ and $\tau(F, \eta)$. That is, when $\delta c_{A}+\gamma c_{I}-t<d^{S}$, the merchant should have an incentive to undertake the credit card business instead of "outsourcing" such a business to the acquirer and the issuer. Since $c_{A}+c_{I}<d^{S}$, as discussed in Section 3, the fixed fee $F$, the interest rate $t$, the network density $\eta_{M}$, and parameters $\delta$ and $\gamma$ play important roles in affecting the merchant's willingness to hold the credit 


\begin{tabular}{|c|c|c|}
\hline Coalition & Characteristic Value & Remark \\
\hline$v(M I)$ & $\begin{aligned} v(M I)= & F+\frac{1+\tau(F, \eta)\left(t-c_{I}-\delta c_{A}\right)}{4} \\
& \times\left[\frac{\alpha^{2}}{\beta}+\frac{\beta c^{2}}{\left[1-\tau(F, \eta) f^{M I}\right]^{2}}\right]-\frac{\beta c}{2} \\
& \times\left[\frac{\alpha}{\beta}+\frac{\tau(F, \eta) c\left(f^{M I}+t-c_{I}-\delta c_{A}\right)}{\left[1-\tau(F, \eta) f^{M I}\right]^{2}}\right]\end{aligned}$ & $\begin{array}{l}\text { The issuer operates for the acquiring } \\
\text { business in addition to its own issuing } \\
\text { business; and the merchant operates } \\
\text { for the retailing business. The interchange } \\
\text { fee rate } f^{M I} \text { results from the negotiation } \\
\text { between the issuer and the merchant. } \\
\text { Example: Sears }(M) \text { and Citibank }(I) / \\
\text { Citibank credit cards }\end{array}$ \\
\hline$v(M A)$ & $\begin{aligned} v(M A)= & F+\frac{1+\tau(F, \eta)\left(t-c_{A}-\gamma c_{I}\right)}{4} \\
& \times\left[\frac{\alpha^{2}}{\beta}+\frac{\stackrel{\beta}{\beta} c^{2}}{\left[1-\tau(F, \eta) d^{M A}\right]^{2}}\right]-\frac{\beta c}{2} \\
& \times\left[\frac{\alpha}{\beta}+\frac{\tau(F, \eta) c\left(d^{M A}+t-c_{A}-\gamma c_{I}\right)}{\left[1-\tau(F, \eta) d^{M A}\right]^{2}}\right]\end{aligned}$ & $\begin{array}{l}\text { The acquirer operates for the issuing } \\
\text { business in addition to its own acquiring } \\
\text { business; and the merchant operates for } \\
\text { the retailing business. The merchant } \\
\text { discount rate } d^{M A} \text { results from the negotiation } \\
\text { between the acquirer and the merchant. } \\
\text { Example: Wal-Mart }(M) \text { and GE Money Bank }(A) \\
\text { /Wal-Mart Discover credit cards }\end{array}$ \\
\hline$v(M A I)$ & $\begin{aligned} v(M A I)= & F+\left(\alpha-\frac{\beta c}{1-\tau(F, \eta) d}\right)\left[\frac{\tau(F, \eta)\left(t-c_{I}-c_{A}\right)}{4}\right. \\
& \left.\left.+\frac{1}{4}\right)\left(\frac{\alpha}{\beta}+\frac{c}{1-\tau(F, \eta) d}\right)-\frac{c}{2}\right]\end{aligned}$ & $\begin{array}{l}\text { The merchant, the acquirer, and the issuer } \\
\text { operate for the retailing, the acquiring, } \\
\text { and issuing business, respectively. } \\
\text { Example: Wal-Mart }(M) \text {, GE Money Bank }(A) \text {, } \\
\text { and Citibank }(I) \text { /Citibank credit cards }\end{array}$ \\
\hline
\end{tabular}

Table 1: The characteristic values $v(M), v(M I), v(M A)$, and $v(M A I)$ for the three-player cooperative game. Note that, in the characteristic value $v(M)$, the retail price in the two-stage game setting is $p^{S}=\left\{\alpha / \beta+c /\left[1-\tau(F, \eta) d^{S}\right]\right\} / 2$ and the retail price in the coalition $\{M\}$ is $p^{M_{1}}=\{\alpha / \beta+c /[1-$ $\left.\left.\tau\left(F, \eta_{M}\right)\left(\delta c_{A}+\gamma c_{I}-t\right)\right]\right\} / 2$.

card business.

When the values of $F$ and $t$ are given, we find that, if the merchant is sufficiently efficient in the credit card business such that $\delta c_{A}+\gamma c_{I}-t<d^{S}$, then the merchant should be inclined to operate the credit card business rather than to subcontract the financial service out to the acquirer and the issuer. Noting that, in practice, the majority of merchants are specialized in the retailing service rather than the financial service, we conclude that the large-scale merchants - which include, e.g., JUSCO, as an example for the coalition $\{M\}-$ may be likely to consider the credit card business, whereas the other merchants (especially, small-scale merchants) may have to rely on the acquirer and the issuer as in the two-stage game setting. Note that the "large" merchants are defined as those who can operate with sufficiently small values of $\delta$ and $\gamma$; and the "small" merchants are those with large values of $\delta$ and $\gamma$.

We also note that small merchants may increase the fixed fee $F$ and the interest rate $t$, in order to act alone in credit card transactions without cooperating with the acquirer and the issuer in the traditional setting. Specifically, if the merchant charges the credit card consumers a higher interest rate, then the retail price $p^{M_{1}}$ can be reduced and the condition that $\delta c_{A}+\gamma c_{I}-t<d^{S}$ may be still satisfied even if $\delta$ and $\gamma$ are not sufficiently small. Moreover, the merchant can increase the fixed fee $F$ - which also results in an increase in its retail price, assuring that $\Pi_{M_{1}}\left(p^{M_{1}}\right) \geq \pi_{M}\left(p^{S}\right)$. Hence, to deal with the credit card operations, the small merchants may need to raise the fixed fee and the interest rate, and also to reduce its retail price to compensate credit card consumers for their higher credit card costs. 
In addition, the negotiated interchange fee rate $f^{M I}$ in the coalition $\{M I\}$ and the negotiated merchant discount rate $d^{M A}$ in the coalition $\{M A\}$ are smaller than the Stackelberg equilibrium-based

merchant discount rate $d^{S}$ in the two-stage game setting. Our analysis in online Appendix $\mathrm{C}$ indicates that $f^{M I} \geq c_{I}+\delta c_{A}-t$ and $d^{M A} \geq c_{A}+\gamma c_{I}-t$.

\subsection{The Analysis of the Three-Player Cooperative Game for the Rates Assuring the Stability of the Grand Coalition}

We use the solution concepts of Shapley value and the nucleolus to find a unique interchange fee rate and a unique merchant discount rate that result in a fair scheme of allocating the profit $v(M A I)$ among the three players. Before we calculate the rates, we first investigate whether our cooperative game in the characteristic-function form is superadditive and convex.

A three-player cooperative game $\mathcal{G}$ is superadditive if $v\left(C_{1} \cup C_{2}\right) \geq v\left(C_{1}\right)+v\left(C_{2}\right)$ for any two disjoint coalitions $C_{1}$ and $C_{2}$ [40]. Moreover, the game is convex and superadditive if its characteristic function is supermodular (Driessen [14] and Topkis [43]). For our problem, the characteristic function of our three-player cooperative game is supermodular if $v(S \cup T)+v(S \cap T) \geq v(S)+v(T)$, for all $S, T \subseteq N[38]$.

\subsubsection{Supermodularity and the Core}

We begin by deriving a sufficient condition under which our cooperative game is supermodular.

Theorem 3 If the merchant discount rate $d$ is negotiated such that $v(M A I)-v(M A) \geq v(M I)-v(M)$, then our three-player cooperative game is supermodular.

In the sufficient condition given by the above theorem, $v(M I)-v(M)$ means the "additional" profit generated when the issuer participates in the credit card business by undertaking both the issuer- and the acquiring-related operations. Moreover, $v(M A I)-v(M A)$ represents the "additional" profit generated by the participation of the issuer who only operates the issuer-related business while the acquirer exists in the credit card network. That is, the sufficient condition in Theorem 3 implies that the issuer, the acquirer, and the merchant can jointly achieve a higher profit from the credit card business (i.e., the game is supermodular and thus superadditive), if the issuer's "contribution" is greater when the acquirer serves the credit card network than when the acquirer is not involved in such a business. Furthermore, one may note that the sufficient condition that $v(M A I)-v(M A) \geq v(M I)-v(M)$ can be equivalently re-written as $v(M A I)-v(M I) \geq v(M A)-v(M)$. Using our above argument, we can also conclude that the three firms can enjoy a higher system-wide profit, if the acquirer's contribution is greater when the issuer exists in the credit card network than when the issuer is not involved.

In addition to the above, we find that the merchant should be always involved in the credit card business, even though both the issuer and the acquirer are important to the profitability — and efficiency — of the credit card network. The reason is given as follows: if the merchant does not join the network, then both the issuer and the acquirer cannot gain any profit from the credit card business since they, as two financial firms, could not play as the role of an "merchant" to realize the sale revenue in the retailing market. 
Remark 2 The merchant's participation in the credit card network is significantly important to the success of the network. Moreover, the issuer and the acquirer may need to have sufficient expertise in the issuing- and the acquiring-related business, respectively, in order to make the network-wide profit higher than when the merchant operates the credit card business by itself or delegates the business to either the issuer or the acquirer. Theorem 3 implies that the issuer and the acquirer should both operate in the credit card network, in order to improve the profitability of the network. That is, the popularity of the credit card service in the financial market could be important to the success of the recent U.S. bill "H.R. 2695," in which the merchant is encouraged to participate in the credit card network for the negotiation of the interchange fee rate and the merchant discount rate.

When our cooperative game is supermodular, it has a non-empty core in which there exists a set of fair allocation schemes each assuring the stability of the grand coalition $\{M A I\}[16]$. Letting $y_{i}$ denote the profit allocated to firm $i, i \in N=\{M, A, I\}$, we can call a proper allocation scheme $y \equiv\left(y_{M}, y_{A}, y_{I}\right)$-where $y_{M}=\Pi_{M_{4}}(d), y_{A}=\Pi_{A_{4}}(d)$, and $y_{I}=\Pi_{I_{4}}(f)$-an imputation for our game $\mathcal{G}=(N, v()$.$) , if the scheme satisfies the following two properties: (i) individual rationality, i.e.,$ $y_{i} \geq v(i)$, for all $i \in N$; and (ii) collective rationality, i.e., $\sum_{i \in N} y_{i}=v(M A I)$ [40]. The core is the set of all undominated imputations (fair allocation schemes) $\left(y_{M}, y_{A}, y_{I}\right)$ such that $\sum_{i \in T} y_{i} \geq v(T)$ for all coalitions $T \subseteq N=\{M, A, I\}$.

Even though a supermodular game must have a non-empty core, we cannot conclude that the core is empty for the non-supermodular game. In the following theorem, we provide necessary and sufficient conditions under which our cooperative game has a non-empty core.

Theorem 4 The core of our cooperative game is non-empty if and only if $f^{M I}-\delta c_{A} \leq f$ and $f+$ $d^{M A}+t-\gamma c_{I} \leq d \leq \min \left(d^{M A}, f^{M I}\right)$.

The above theorem implies that, as the merchant joins the grand coalition $\{M A I\}$ and bargains with the acquirer and the issuer over the merchant discount rate and the interchange fee rate, the negotiated interchange fee rate and merchant discount rate are smaller than $d^{M A}$ and $f^{M I}$, respectively. Noting from Section 4.1 that both $d^{M A}$ and $f^{M I}$ are smaller than $d^{S}$, we find that the merchant discount rate negotiated by the issuer, the acquirer, and the merchant is smaller than $d^{S}$ in the two-stage game setting. But, we cannot analytically show that the interchange fee rate is smaller than $f^{*}$ in the twostage game setting. Though, all numerical examples that we shall provide later can demonstrate that the interchange fee rate is reduced as a result of the negotiation among the issuer, the acquirer, and the merchant.

As Theorem 4 indicates, the interchange fee rate $f$ must be greater than or equal to $f^{M I}-\delta c_{A}$. We learn from Section C.2 that $f^{M I}$ is the rate paid by the merchant to the issuer in the coalition $\{M I\}$, where the issuer absorbs the unit $\operatorname{cost} \delta c_{A}$ in undertaking the acquiring-related operations. This implies that $f^{M I}-\delta c_{A}$ represents the issuer's unit "net gain" resulting from its own (issuer-related) operations. Thus, the condition that $f^{M I}-\delta c_{A} \leq f$ assures that the issuer is willing to join the grand coalition $\{M A I\}$. Similarly, in the two-player coalition $\{M A\}$, the acquirer's unit "net gain" resulting from its own (acquiring-related) operations is $d^{M A}+t-\gamma c_{I}$. To assure that the acquirer has an incentive to join the grand coalition $\{M A I\}$, we should determine the merchant discount rate $d$ such that the 
acquirer's unit net gain $(d-f)$ in $\{M A I\}$ is no less than $d^{M A}+t-\gamma c_{I}$, i.e., $f+d^{M A}+t-\gamma c_{I} \leq d$. Note that the merchant pays the rates $d^{M A}$ and $f^{M I}$ in the two-player coalitions $\{M A\}$ and $\{M I\}$, respectively. In order to entice the merchant to stay in the grand coalition, we should choose the merchant discount rate that is lower than both $d^{M A}$ and $f^{M I}$, i.e., $d \leq \min \left(d^{M A}, f^{M I}\right)$. According to the above discussion, we draw the following insights.

Remark 3 The grand coalition is more likely to be stable, if the degree of "division of labour" among the three firms (i.e., the merchant, the acquirer, or the issuer) in the credit card network is higher. The division of labour is the specialization of cooperative "labour" in specific, circumscribed tasks, see, e.g., [41]. In our credit card network, the degree of division of labour reflects the specializations of the merchant, the acquirer, or the issuer in their retailing, acquiring, and issuing operations, respectively. When the degree of division of labour in the network is higher, each firm is stronger and more "specialized" in its own operation; that is, the acquirer and the merchant in the coalition $\{M A\}$ may operate the credit card issuing business more inefficiently, and the issuer and the merchant in the coalition $\{M I\}$ may operate the credit card acquiring business more inefficiently. Note that the scale parameter $\delta$ reflects the operation efficiency of the issuer and the merchant in acting as an "acquirer;" and, the scale parameter $\gamma$ reflects the operation efficiency of the acquirer and the merchant in acting as an "issuer." Therefore, a higher degree of division of labour should result in higher values of $\delta$ and $\gamma$.

Theorem 4 indicates that, in the credit card network, all of the three firms are willing to join the grand coalition, if and only if the deviation of any one player from the grand coalition shall make the remaining two players worse off. That is, if each two-player coalition can achieve a significantly high profit (characteristic value), then the grand coalition is unlikely to be stable. Recall that the profit of the coalition $\{A I\}$ is zero. From online Appendices C.2 and C.3, we find that the profits of the coalitions $\{M I\}$ and $\{M A\}$ could be small if the values of the scale parameters $\delta \geq 1$ and $\gamma \geq 1$ are significantly high, respectively. Therefore, in the credit card network with the high values of $\delta$ and $\gamma$, neither the two-player coalition $\{M I\}$ nor $\{M A\}$ would be stable and instead, the grand coalition is likely to be stable. That is, if the merchant, the acquirer, and the issuer are more specialized in their own operations for the credit card business - resulting in a higher degree of division of labour, then it should be more costly for the issuer and the merchant (the acquire and the merchant) to take the role of an "acquirer" ("issuer"), the value of $\delta(\gamma)$ is higher, and the grand coalition is more likely to be stable.

The above remark implies that the recent U.S. bill "H.R. 2695" should be more effective, if, in major U.S. credit card networks, the degree of division of labour is higher, and the financial firms and the merchants are more specialized in their own operations. As discussed in Section 3, in 2007, the percentage of the transactions by the financial firms mainly acting for the acquiring operation is around $79.9 \%$, and the percentage of the transactions by the financial firms mainly acting in the issuing operation is around $83.7 \%$. Moreover, there is no merchant on either the list of top 10 acquirers or the list of top 10 issuers, which was listed by Prager et al. [32]. The above fact implies that the degree of division of labour in the U.S. credit card business is high. Thus, we can conclude that the recent U.S. bill "H.R. 2695" should be effective as merchants, acquirers, and issuers should be willing to form a 
stable grand coalition and negotiate the merchant discount rate and the interchange fee rate.

In addition, we find from Theorem 4 that a low value of the interest rate $t$ is likely to result in the stability of the grand coalition and the effectiveness of the U.S. bill "H.R. 2695." Letting

$$
\Theta \equiv\left\{(d, f) \mid f+d^{M A}+t-\gamma c_{I} \leq d \leq \min \left(d^{M A}, f^{M I}\right) \text { and } f^{M I}-\delta c_{A} \leq f\right\}
$$

we have the following corollary.

Corollary 1 If $\gamma>1$, then the set $\Theta$ is non-empty and thus, the interchange fee rate and the merchant discount rate in $\Theta$ can assure that the corresponding allocation scheme is in the core and the grand coalition $\{M A I\}$ is stable. However, if $\gamma=1$, then both the set $\Theta$ and the core are empty.

In this paper, $\gamma$ is assumed to be greater than 1, because, as discussed previously, the acquirer/merchant incurs a higher operation cost in acting as "an issuer." Moreover, noting from Section 4.1 that $d^{M A}<d^{S}$ and $f^{M I}<d^{S}$, we find that $d^{S}>\min \left(d^{M A}, f^{M I}\right)$ and thus, the rates $\left(f^{*}, d^{S}\right)$ in the two-stage game (traditional) setting - which are given as in Section 3-do not belong to the set $\Theta$. That is, if the merchant, the acquirer, and the issuer choose the rates $\left(f^{*}, d^{S}\right)$ in the three-player cooperative game setting, then the core is empty and the grand coalition is not stable.

\subsubsection{The Unique Merchant Discount and Interchange Fee Rates}

Since the core includes many allocation schemes rather than only a unique scheme, one may need to make a decision on which imputation in the core to be chosen to allocate the profit $v(M A I)$. Shapley value [37] and the nucleolus [35] are the two most commonly-used solutions in cooperative game theory. We thus apply both of them to derive a unique allocation solution and its corresponding rates $d$ and $f$. Moreover, the three players may desire to choose the globally-optimal rates that maximize their total profit $v(M A I)$. Accordingly, in addition to Shapley value and nucleolus, we also compute the globally optimal rates.

Shapley value For a $n$-player cooperative game, the Shapley value for player $i$ is computed as $\phi_{i}=\sum_{i \in T}(|T|-1) !(n-|T|) ![v(T)-v(T-\{i\})] /(n !)$, where $T$ denotes a possible coalition that the firm $i$ joins, and $|T|$ is the size of $T$. It is a unique, monotonic solution (Megiddo [28] and Young [49]), as the profit allocated to each player increases if the profit achieved by each possible coalition increases.

Using the above formula, we can write the unique allocation scheme in terms of Shapley value for our cooperative game $\mathcal{G}=(N, v(\cdot))$ as,

$$
\left\{\begin{aligned}
\phi_{M} & =\frac{2 v(M A I)+2 v(M)+v(M A)+v(M I)}{6} \\
\phi_{A} & =\frac{2 v(M A I)-v(M)+v(M A)-2 v(M I)}{6} \\
\phi_{I} & =\frac{2 v(M A I)-v(M)+v(M I)-2 v(M A)}{6}
\end{aligned}\right.
$$

Note that $\phi_{M}, \phi_{A}$, and $\phi_{I}$ correspond to the profits allocated to the merchant, the acquirer, and the issuer in the grand coalition $-\Pi_{M_{4}}(d), \Pi_{A_{4}}(d)$, and $\Pi_{I_{4}}(f)$ given in (17) and (18), respectively. By 
solving the equations $\left\{\Pi_{M_{4}}(d)=\phi_{M}\right.$ and $\left.\Pi_{A_{4}}(d)=\phi_{A}\right\}$, we can find the Shapley value-characterized merchant discount rate $d^{S P}$ and interchange fee rate $f^{S P}$. Note that we do not need to consider the equation that $\Pi_{I_{4}}\left(f^{S P}\right)=\phi_{I}$, because $\Pi_{M_{4}}\left(d^{S P}\right)+\Pi_{A_{4}}\left(d^{S P}\right)+\Pi_{I_{4}}\left(f^{S P}\right)=\phi_{M}+\phi_{A}+\phi_{I}=v(M A I)$. However, the Shapley value-characterized $d^{S P}$ and $f^{S P}$ may not belong to the set $\Theta$; thus, when $d^{S P}$ and $f^{S P}$ are adopted, the core may be empty and the grand coalition $\{M A I\}$ may be unstable.

To illustrate the above, we next provide two examples - one with an empty core and the other with a non-empty core - when the Shapley value is used to allocate $v(M A I)$ among the merchant, the acquirer, and the issuer.

Example 2 We consider a three-product case and use the parameter values given in Example 1. We also assume that $\delta=15$ and $\gamma=12$. In addition, for the coalition $\{M\}$, the density of the merchant's network in (9) is $\eta_{M}=0.5$. According to Section 4.1, we find that (i) for the coalition $\{M\}$, $v(M)=51.44$; (ii) for the coalition $\{M I\}, f^{M I}=6.3 \%$ and $v(M I)=88.70$; and (iii) for the coalition $\{M A\}, d^{M A}=6.2 \%$ and $v(M A)=69.24$. Solving the equation set $\left\{\Pi_{M_{4}}(d)=\phi_{M}\right.$ and $\left.\Pi_{A_{4}}(d)=\phi_{A}\right\}$ yields $d^{S P}=2.75 \%$ and $f^{S P}=0.07 \%$, and $v(M A I)=101.38$. Since $f^{S P}<f^{M I}-\delta c_{A}=0.3 \%$, $\left(d^{S P}, f^{S P}\right)$ is not in the set $\Theta$. Therefore, as Corollary 1 indicates, the core is empty and the grand coalition $\{M A I\}$ is thus unstable. $\triangleleft$

We then provide another example to calculate the Shapley value-based rates that can result in the stability of the grand coalition.

Example 3 We re-consider Example 2 but assume that $c_{A}=0.004, c_{I}=\$ 0.0045$ and $\gamma=15$. Similarly, we find that $v(M)=48.69 ; v(M I)=68.97$, and $v(M A)=65.31 ;$ and, $f^{M I}=6.25 \%$ and $d^{M A}=6.95 \%$. We then obtain the rates $d^{S P}=2.99 \%$ and $f^{S P}=0.33 \%$. It follows that $v(M A I)=101.57$. Since $f^{S P}+d^{M A}+t-\gamma c_{I}=0.73 \% \leq d^{S P} \leq \min \left(d^{M A}, f^{M I}\right)=6.25 \%$ and $f^{S P}>f^{M I}-\delta c_{A}=0.25 \%,\left(d^{S P}, f^{S P}\right)$ belongs to the set $\Theta$ and thus, the core is non-empty. That is, if the merchant, the acquirer, and the issuer choose $d^{S P}$ and $f^{S P}$, then the grand coalition $\{M A I\}$ is stable and the three players are willing to cooperate for the credit card business. $\triangleleft$

The Nucleolus Since the Shapley value-characterized rates $\left(d^{S P}, f^{S P}\right)$ may not be in the set $\Theta$, we use another common concept "the nucleolus" to find a unique pair of the rates. For a three-player cooperative game, the nucleolus solution is defined as a 3-tuple imputation $\mathbf{x}=\left(x_{M}, x_{A}, x_{I}\right)$ such that the excess ("unhappiness") $e_{S}(x)=v(S)-\sum_{i \in S} x_{i}$ of any possible coalition $S$ cannot be lowered without increasing any other greater excess [35]. That is, the nucleolus solution makes the largest unhappiness of the coalitions as small as possible, or, equivalently, minimizes the worst inequity. A most common approach to find the nucleolus is the sequential linear programming (LP) method that is based on lexicographic ordering (Maschler, Peleg, and Shapley [26]). Using the LP approach, we first reduce the largest excess $\max \left\{e_{S}(x)\right.$, for all $\left.S \subseteq N\right\}$ as much as possible, then decrease the second largest excess as much as possible, and continue this process until the 3-tuple imputation $\mathbf{x}$ is determined. Even though the nucleolus has been proved to always exist in the core as long as the core is non-empty [40], we have to solve a series of linear problems to find it. For a recent detailed discussion on the calculation of the nucleolus, see Leng and Parlar [22]. For our game, a LP algorithm can be 
developed as given in online Appendix D. We next provide two examples to illustrate the calculation of the nucleolus-characterized rates $d^{n}$ and $f^{n}$.

Example 4 We consider the parameter values given in Example 2. Using the algorithm provided in online Appendix D, we compute the nucleolus-characterized rates as $\left(d^{n}, f^{n}\right)=(3.53 \%, 0.45 \%)$, which can result in the stability of the grand coalition. Next, we adopt the parameter values used in Example 3 to find the nucleolus-characterized rates as $d^{n}=3.58 \%$ and $f^{n}=0.44 \%$. Comparing the nucleolus-characterized solution $\left(d^{n}, f^{n}\right)$ with the Shapley value-characterized solution $\left(d^{S P}, f^{S P}\right)=$ $(2.99 \%, 0.33 \%)$ in Example 3, we find that the nucleolus solution suggests a higher merchant discount rate and also a higher interchange fee rate. Since $d^{n}-f^{n}=3.14 \%>d^{S P}-f^{S P}=2.66$, both the acquirer and the issuer should prefer the nucleolus to the Shapley value. $\triangleleft$

Globally-Optimal Rates We now investigate a centralized case that the merchant, the acquirer, and the issuer jointly determine optimal merchant discount and interchange fee rates to maximize their total profit $v(M A I)$, which is given as in (19). We note that $v(M A I)$ is dependent on the merchant discount rate $d$ but independent of the interchange fee rate $f$, which is attributed to the fact that the interchange fee - calculated as $f \times \tau(F, \eta) \times R$-is the issuer's revenue but is the acquirer's cost. But, to find the globally optimal merchant discount rate, we should maximize $v(M A I)$ subject to $d \in \Theta$, where $\Theta$ is dependent on the interchange fee rate $f$, as defined in (10). Note that the constraint $d \in \Theta$ is imposed to assure the stability of the grand coalition $\{M A I\}$.

Theorem 5 Given the interchange fee rate $f$, the globally optimal $d^{G}(f)$ maximizing $v(M A I)$ subject to $d \in \Theta$ is obtained as $d^{G}(f)=f+d^{M A}+t-\gamma c_{I}$.

From the above theorem, we learn that, when the three players decide to maximize their total profit $v(M A I)$ for a given value of $f$, then the globally optimal merchant discount rate $d^{G}$ must be no greater than the nucleolus-characterized rate $d^{n}$, i.e., $d^{G} \leq d^{n}$, because $d^{G}(f)=f+d^{M A}+t-\gamma c_{I} \leq d^{n}$. Since the interchange fee rate $f$ is negotiated between the acquirer and the issuer, the optimal rate $f^{G}$ can be obtained by solving the following constrained maximization problem: $\max _{f \geq f^{M I}-\delta c_{A}} \Lambda^{G}(f)=$ $\Pi_{A_{4}}\left(d^{G}(f)\right) \times \Pi_{I 4}(f)$. Similar to Theorem $2, \Lambda^{G}(f)$ can be shown to be a quasi-concave function. But, due to the intractable complexity, we cannot find the closed-form solution for $f^{G}$ but instead write $f^{G}=\arg \max _{f \geq f^{M I}-\delta c_{A}} \Lambda^{G}(f)$. As a result, the globally optimal merchant discount rate $d^{G}=$ $d^{G}\left(f^{G}\right)=f^{G}+d^{M A}+t-\gamma c_{I}$.

Example 5 We first use the parameter values given in Example 2-where the rates in terms of Shapley value cannot assure the stability of the grand coalition - to compute the globally optimal rates as $\left(d^{G}, f^{G}\right)=(0.6 \%, 0.3 \%)$. Next, using the parameter values used in Example 3, we find $\left(d^{G}, f^{G}\right)=$ $(0.65 \%, 0.25 \%)$. We learn from the above results that, in order to maximize $v(M A I)$, the three firms should reduce the globally-optimal interchange fee and merchant discount rates to $f^{M I}-\delta c_{A}$ and $f^{M I}+d^{M A}+t-\delta c_{A}-\gamma c_{I}$, respectively. In fact, we find that such a result holds for a large number of numerical examples. $\triangleleft$ 


\section{$5 \quad$ Further Discussions}

In this section, we investigate a two-player case for a credit card network where the acquirer and the issuer are the same firm, and examine the robustness of our results (obtained in Sections 3 and 4) when the linear price-dependent demand function (specified in Section 3.1.1) is replaced with a logistic function.

\subsection{Discussion on Implications for the Two-Player Games with a Single Firm Act- ing as Both the Acquirer and the Issuer}

We investigate a two-player case for a credit card network where the acquirer and the issuer are the same firm. We learn from Prager et al. [32] that, even though most of top acquirers and issuers are different firms, some firms (e.g., Bank of America) still operate for both the acquiring and the issuing business. Motivated by this practice, we now assume that a single firm acts as both the acquirer and the issuer, and discuss possible changes on the retail price and the merchant discount rate.

When a single financial firm takes both the acquiring and the issuing operations, there is no interchange fee because such a fee is a side payment from an acquirer to an issuer only when the acquirer and the issuer are separate firms in the credit card business. Thus, for a credit card network involving a merchant and a single financial firm, we should only need to determine the merchant's retail price $p$ and the merchant discount rate $d$. Similar to Section 3, we also consider the traditional setting to analyze a non-cooperative game where the merchant does not bargain with the financial firm over the merchant discount rate; and, similar to Section 4, we consider a two-player cooperative game where the merchant negotiates the rate $d$ with the financial firm. Note that the analysis of the above games should be much simpler than that when three players (the merchant, the acquirer, and the issuer) operate in the credit card network. Hence, for the two-player case, we do not solve the two games but instead discuss the implications when a single firm acts for both the acquiring and the issuing business.

In the traditional setting where the merchant does not negotiate with the financial firm about the value of $d$, the credit card operation can be described as a sequential game where the financial firm determines and announces the rate $d$ as a leader and the merchant then responds as a follower by making its retail pricing decision. This game is very similar to the sequential game in the second stage of our two-stage game problem in Section 3. Specifically, for the two-player case, the merchant's bestresponse pricing analysis is the same as that in Section 3.1.1. However, the single financial firm's profit function should be written as $F+\left(d+t-c_{I}-c_{A}\right) \tau(F, \eta) R(d)$, which is similar to the acquirer's profit in (5) for the three-player case involving the interchange fee rate $f>c_{I}$. We learn from Theorem 1 that the optimal merchant discount rate is increasing in $f$. This implies that, in Stackelberg equilibrium, the merchant discount rate for the two-player case should be smaller than that for the three-player case; and, the retail price is also smaller in the two-player case. The result reflects the fact that the financial firm's cost is reduced as a result of the absence of the interchange fee, and the firm and the merchant can thus decrease their merchant discount rate and retail price to increase the sales and improve their profitability.

In the cooperative game setting where the merchant and the financial firm negotiate the rate $d$. 
Different from our three-player cooperative game in Section 3, we should need to investigate the twoplayer cooperative game using the concept of NBS [29]. Such an analysis is very similar to our analysis for the coalition $\{M A\}$ in the three-player cooperative game, which is given in online Appendix C.3. Specifically, we can easily find the result for the two-player cooperative case by simply replacing the parameter $\gamma$ for the coalition $\{M A\}$ in the three-player case with the value of 1 . Similarly using our argument in online Appendix C.3, we can conclude that, for the two-player case, the merchant discount rate in the cooperative game setting is not higher than that in the non-cooperative (sequential) game setting.

Recall from Theorem 4 that, for the three-player case, the merchant discount rate that is negotiated among the merchant, the acquirer, and the issuer is no less than $f+d^{M A}+t-\gamma c_{I}$ but no greater than $\min \left(d^{M A}, f^{M I}\right)$, assuring the stability of the grand coalition. Similar to online Appendix C.3, we can find that the merchant discount rate for the two-player case is greater than $c_{A}+c_{I}-t$, which is smaller than $f+d^{M A}+t-\gamma c_{I}$ as shown in the proof of Theorem 5. However, we cannot immediately conclude that, when the merchant bargains over $d$ with the financial firm for the two-player case, the resulting merchant discount rate is smaller than or greater than that in the three-player case.

The above indicates that, when a single financial firm performs both the acquiring and the issuing tasks in a credit card network, the participation of the merchant for the rate negotiation - which is in gear with the recent U.S. bill "H.R. 2695" - will result in a decrease in the merchant discount rate. Next, we provide an example to illustrate our above argument.

Example 6 We consider the parameter values in Example 1. For the two-stage game where the merchant discount rate is determined by the financial firm rather than negotiated by the merchant and the financial firm, we substitute the merchant's sales revenue $R(d)$ in $(3)$ into the financial firm's profit function $F+\left(d+t-c_{I}-c_{A}\right) \tau R(d)$, and maximize it subject to $d \leq \kappa$ where $\kappa$ is given as in (2). The Stackelberg equilibrium-characterized merchant discount rate for the two-player setting can be obtained as $d^{S}=13.77 \%$, which is smaller than the merchant discount rate $15.56 \%$ in Example 1 where the acquirer and the issuer are two separate firms. The result is the same as our above discussion. We calculate the merchant discount rate that is negotiated by the merchant and the financial firm. Similar to our calculation for the coalition $\{M A\}$, we find that the NBS-characterized rate is $4.90 \%$, which is significantly smaller than the above Stackelberg equilibrium $d^{S}=13.77 \%$. This important result is the same as that in the three-player setting.

Next, we consider another example using the parameter values in Example 2. We find that the Stackelberg equilibrium $d^{S}=13.75 \%$, which is smaller than the equilibrium (i.e., 15.53\%) in the threeplayer setting. Moreover, we find that the NBS-based rate is $4.87 \%$, which is much smaller than the Stackelberg equilibrium.

The above numerical results demonstrate that for the non-cooperative case, the Stackelberg equilibriumbased merchant discount rate in the two-player setting is smaller than that in the three-player setting, whereas for the cooperative case, the negotiated merchant discount rate in the two-player setting is greater than that in the three-player setting. $\triangleleft$ 


\subsection{Robustness of Major Results in the Presence of a Logistic Demand Function}

We now examine whether or not our major results obtained in Sections 3 and 4 still hold, if we relax our assumption on the linearity of the price-depedent demand function and instead consider the following logistic demand function (see, e.g., Phillips [31]):

$$
q^{\prime}(p)=B \times \frac{1}{1+\exp \left(\alpha^{\prime}+\beta^{\prime} p\right)},
$$

where $B$ represents the market size, and $\alpha^{\prime}, \beta^{\prime}>0$ denote the price-independent and the pricedependent parameters, respectively. Since both the two-stage and the cooperative game analyses with the demand function in (11) are intractable, we perform subsequent numerical experiments with the parameter values in (11) as $B=200, \alpha^{\prime}=0.03$, and $\beta^{\prime}=0.07$. The values of other parameters are the same as those in Examples 1 and 2.

\subsubsection{The Numerical Analysis for the Two-Stage Game with the Logistic Demand Func- tion}

We first examine whether or not our results in Section 3 hold when the price-dependent demand function is given as in (11). Recall from Section 3.1.1 that the merchant should compare its profits $\pi_{M}^{N C}(p)$ and $\pi_{M}^{C}(p)$ in (1) to determine if consumers are allowed to shop with the credit card issued by the issuer. To entice the merchant to accept the credit card, the acquirer should announce its merchant discount rate $d$ such that $\pi_{M}^{C}(p) \geq \pi_{M}^{N C}(p)$. Noting that $\pi_{M}^{C}(p)$ is decreasing in $d$ and $\pi_{M}^{N C}(p)$ is independent of $d$, we find that there must exist a cutoff level $\kappa$-which can be specified as in (2) when the demand is linear - such that the merchant benefits from accepting credit card transactions and thus agrees on the use of the credit card, if the merchant discount rate $d$ is no greater than the cutoff level $\kappa$. The above calculation implies that the result in Section 3.1.1 holds when the demand function is specified as in (11).

Next, we examine the result in Remark 1. Using the values of parameters in Example 1, we compute the interchange fee rate negotiated between the acquirer and the issuer as $f^{*}=5.95 \%$, the Stackelberg equilibrium-based merchant discount rate and retail price as $d^{S}=17.00 \%$ and $p^{S}=\$ 148.73$. When the interchange fee rate is determined by the issuer itself, we can calculate the resulting rate as $f^{*}=$ $d^{S}=\kappa=30.03 \%$, which means that the acquirer's profit margin is zero. The above result implies that Remark 1 holds when the demand function is given as in (11).

\subsubsection{The Numerical Analysis for the Cooperative Game with the Logistic Demand Function}

We begin by examining the result in Section 4.1. Using the values of parameters in Example 2, we find that $\delta c_{A}+\gamma c_{I}-t=0.118<d^{S}=0.17$. We calculate the merchant's maximum profit in the two-stage game setting and that in the coalition $\{M\}$ as $\pi_{M}\left(p^{S}\right)=\$ 7.44$ and $\Pi_{M_{1}}\left(p^{M_{1}}\right)=\$ 16.89$. When we increase the value of $\delta$ from 15 to 20 and the value of $\gamma$ from 12 to 15 , and decrease the value of $F$ from 15 to 10 , we find that $\delta c_{A}+\gamma c_{I}-t=0.153>d^{S}=0.082$, and $\pi_{M}\left(p^{S}\right)=\$ 12.66>\Pi_{M_{1}}\left(p^{M_{1}}\right)=\$ 11.08$. 
The above results imply that, if the values of $\delta$ and $\gamma$ are higher and/or the value of $F$ is lower, then the merchant is more unlikely to operate the credit card business by itself. That is, our result in Section 4.1 also applies to the game with the logistic demand function as in (11).

Next, we investigate if Theorems 3 and 4 are applicable to the cooperative game with the logistic demand function. The characteristic values of the game are calculated as follows: $v(\varnothing)=v(A)=$ $v(I)=v(A I)=0, v(M)=16.89, v(M I)=41.18, v(M A)=32.90$, and $v(M A I)=97.54$. For the game, the merchant discount and the interchange fee rates in terms of Shapley value are computed as $d^{S P}=6.92 \%$ and $f^{S P}=2.37 \%$. We can find from Driessen [14] and Topkis [43] that the cooperative game is supermodular. Noting that $v(M A I)-v(M A)=64.64>v(M I)-v(M)=24.29$, we conclude that Theorem 3 may hold for the game with the demand function in (11). The core of this cooperative game is thus non-empty. We examine the sufficient and necessary condition in Theorem 4 as follows: $f^{M I}-\delta c_{A}=0.083-15 \times 0.004=0.023 \leq f^{S P}=0.0237$, and $f^{S P}+d^{M A}+t-\gamma c_{I}=0.0427<$ $d^{S P}=0.0692<\min \left(d^{M A}, f^{M I}\right)=0.077$. This means that Theorem 4 also holds for the game with the logistic function. We preform a great number of numerical experiments, and find that Theorems 3 and 4 always apply to our game analysis, which should demonstrate the robustness of the two theorems. In fact, one may note from our discussion in Section 4.2.1 that Theorems 3 and 4 could not largely depend on the linearity property of our demand function.

In addition, we also find that, for most cases, Shapley value cannot assure the stability of the grand coalition. Therefore, we still calculate the nucleolus to represent the negotiated merchant discount and interchange fee rates. For Examples 2 and 3, we use the algorithm provided in online Appendix D to compute the nucleolus-characterized rates $\left(d^{n}, f^{n}\right)$ as $(8.43 \%, 2.60 \%)$ and $(6.86 \%, 1.93 \%)$, respectively. Similar to our discussion in Example 4, we compare the nucleolus $\left(d^{n}, f^{n}\right)=(8.43 \%, 2.60 \%)$ and Shapley value $\left(d^{S P}, f^{S P}\right)=(6.92 \%, 2.37 \%)$, and find that both the acquirer and the issuer should prefer the nucleolus to the Shapley value.

Next, we calculate the globally-optimal rates that maximize the total profit $v(M A I)$. For Examples 2 and 3 , we find that the optimal rates $\left(d^{G}, f^{G}\right)$ as $(4.31 \%, 2.31 \%)$ and $(3.74 \%, 1.12 \%)$, respectively. Our results indicate that Theorem 5 may not hold when the demand function is given in (11), which is mainly attributed to the fact that Theorem 5 results from the linearity of the demand function. However, we find that in the centralized setting, the merchant discount rate and the interchange fee rate are both reduced; this is the same as what we find from Example 5.

We also find that, when a financial firm performs both the acquiring and the issuing operations, for Example 2, the merchant discount rate in Stackelberg equilibrium is $d^{S}=15.22 \%$, which is smaller than the Stackelberg equilibrium-based rate in the three-player setting (17.00\%). In addition, for the cooperative case, the negotiated merchant discount rate in the two-player setting is $12.39 \%$, which is greater than that in the three-player setting (8.43\%). It thus follows that our major results in Section 5.1 hold for the game with the logistic demand function.

Remark 4 From our numerical experiments, we learn that our major managerial insights based on the linearity of the demand function still hold when we use the logistic demand function in (11), even though Theorem 5 does not apply to the game analysis with the logistic demand function. In fact, Theorem 5 is dependent on the linearity property of the demand function; but, the managerial insights 
resulting from Theorem 5 hold because in the centralized setting with the logistic function, both the merchant discount rate and the interchange fee rate are both decreased. In conclusion, we find that our results in Sections 3 and 4 are robust in general. $\triangleleft$

\section{Summary and Concluding Remarks}

This paper considers a credit card network involving an issuer, an acquirer, and a merchant who serves consumers in a consumer market. For the network, we determine the interchange fee rate for the issuer, the merchant discount rate for the acquirer, and the optimal retail price for the merchant in both a twostage game setting (where the merchant does not participate in the negotiation of the interchange fee rate and the merchant discount rate) and a three-player cooperative game setting (where the merchant is involved in the negotiation). For the former setting, we derive the NBS-characterized interchange fee rate and the Stackelberg equilibrium-based merchant discount rate and retail price.

For the cooperative game setting, we calculate the characteristic values of all possible coalitions, derive a sufficient condition for the supermodularity, and attain a necessary and sufficient condition under which the core of our cooperative game is non-empty. The condition for the non-emptiness of the core indicates that the issuer, the acquirer, and the merchant are willing to jointly determine the rates if and only if the interchange fee rate is no smaller than a specific threshold and the merchant discount rate falls in a specific range. We then apply Shapley value, the nucleolus, and the globally-optimal solution to find unique rates at which the merchant, the acquirer, and the issuer are all better off by joining the credit card network. We show that both the nucleolus-characterized and the globallyoptimal rates can assure the stability of the grand coalition, whereas the Shapley value-characterized rates are likely to make the grand coalition unstable. We also discuss the implications for two-player games involving a merchant and a financial firm who acts for both the acquiring and the issuing operations.

Our major managerial insights are summarized as follows:

1. In the two-stage game setting, the acquirer should determine a merchant discount rate that is no larger than a specific cutoff level [i.e., $\kappa$ in (2)], in order to entice the merchant to accept the transactions with credit cards. Otherwise, the merchant may not be willing to participate in the credit card business.

2. In the two-stage game setting, if the interchange fee rate is determined by the issuer itself rather than by the negotiation between the issuer and the acquirer, then the acquirer would not benefit from the credit card business. This implies that such a two-player negotiation can help entice the acquirer to stay in the credit card network.

3. In the cooperative game setting, the "large" merchants (e.g., JUSCO) — which can also efficiently operate the acquiring and the issuing business - may have an incentive to undertake the credit card operations by itself. The other merchants (especially, "small" merchants) may have to increase the fixed fee and the interest rate, if they desire to act for the credit card business by themselves. Otherwise, the small merchants have to only focus on the retailing service, cooperating with the issuer and the acquirer as in the two-stage game setting. 
4. The merchant's participation in the credit card network is significantly important to the profitability of the network. Moreover, the issuer and the acquirer may need to have sufficient expertise in the issuing- and the acquiring-related business, respectively, in order to make the network-wide profit higher than when the merchant operates the credit card business by itself or delegates the business to either the issuer or the acquirer.

Moreover, the popularity of the credit card service in the financial market would be important to the success of the recent U.S. bill "H.R. 2695," in which the merchant is encouraged to participate in the credit card network for the negotiation of the interchange fee rate and the merchant discount rate.

5. The interchange fee rate and the merchant discount rate in the cooperative game setting are smaller than those in the two-stage game setting. This means that, as a result of implementing the U.S. bill "H.R. 2695," both rates are reduced, which shows that the bill should be effective.

6. The grand coalition should be more likely to be stable, if the degree of division of labour in the credit card business is higher and the issuer, the acquirer, and the merchant are all more specialized in their own operations. As discussed in Section 4.2.1, in the United States, the degree of division of labour in the credit card business is high, and thus, the recent U.S. bill "H.R. 2695" should be effective. In addition, a low value of the interest rate $t$ is likely to result in the stability of the grand coalition and the effectiveness of the U.S. bill "H.R. 2695."

In addition, we perform sensitivity analysis to examine the impacts of the credit card operation costs (i.e., $c_{A}, c_{I} ; \delta, \gamma$ ) on the interchange fee rate and the merchant discount rate in the two-stage and the three-player cooperative games. Through a large number of numerical experiments, we find that, for most cases, the Shapley value-characterized rates cannot assure the stability of the grand coalition, and the globally-optimal interchange fee and merchant discount rates are equal to $f^{M I}-\delta c_{A}$ and $f^{M I}+d^{M A}+t-\delta c_{A}-\gamma c_{I}$, respectively. Thus, using the parameter values given in Example 2 as base values, we calculate the Stackelberg equilibrium-based rates - for the two-stage game when the merchant does not bargain over the rates - and the nucleolus-characterized rates - for the three-player cooperative game when the merchant participates in the rate negotiation. We also show that our main managerial results hold for the game with a logistic demand function.

According to our results given in online Appendix E, we find that the interchange fee rate and the merchant discount rate in the cooperative game are significantly smaller than the Stackelberg equilibrium rates in the two-stage game setting. We also learn that the acquirer's and the issuer's unit operation costs $\left(c_{A}\right.$ and $\left.c_{I}\right)$ more significantly impact the interchange fee rate and the merchant discount rate in the cooperative game setting than in the two-stage game setting. The interchange fee rate in the two-stage game setting is decreasing in the acquirer's unit operation cost $c_{A}$, whereas the interchange fee rate in the cooperative game setting may not significantly depend on the value of $c_{A}$ when $c_{A}$ is sufficiently small (i.e., $c_{A}<0.4$ ). But, the merchant discount rate is always increasing in $c_{A}$ in both the two-stage and the cooperative game settings.

In both the two-stage and the cooperative game settings, the interchange fee rate and the merchant discount rate are increasing in the issuer's unit operation cost $c_{I}$. In the cooperative game setting, the interchange fee rate may not significantly depend on the value $\delta$-which measures the per dollar 
operation cost absorbed by the merchant/issuer in acting as an acquirer when $\delta$ is sufficiently small (i.e., $\delta<14$ ); but, the merchant discount rate is increasing in $\delta$. In addition, the parameter $\gamma$-which measures the per dollar operation cost absorbed by the merchant/acquirer in acting as an issuer-may have no significant impact on the interchange fee rate when $\gamma$ is sufficiently small (i.e., $\gamma<11.5$ ).

In conclusion, we find that the recent U.S. bill "H.R. 2695" should be useful to reduce both the interchange fee rate and the merchant discount rate. We have identified some important managerial insights that could help improve the efficiency of the credit card operations.

\section{References}

[1] D. Akers, J. Golter, B. Lamm, and M. Solt. Overview of recent developments in the credit card industry. FDIC Banking Review, 17(3):23-35, 2005.

[2] Association for Financial Professionals. Retail industry forum survey: Consumer payment choices and bank relationships, 2005. www.afponline.ca/WorkArea/DownloadAsset.aspx?id=7526 (URL last accessed on June 12, 2012).

[3] D. Balto. The problem of interchange fees: Costs without benefits? E.C.L.R., pages 215-224, 2000.

[4] W. F. Baxter. Bank interchange of transactional papers: Legal and economics perspectives. Journal of Law \& Economics, XXVI:541-588, October 1983.

[5] J. Bertrand. Théorie mathématique de la richesse sociale. Journal des Savants, pages 499-509, September 1883. Paris.

[6] W. Bolt and S. Chakravorti. Economics of payment cards: A status report. Economic Perspectives, pages 15-27, The 4th Quarter 2008.

[7] A. Brandenburger and H. Stuart. Biform games. Management Science, 53(4):537-549, April 2007.

[8] A. M. Brandenburger and B. J. Nalebuff. Co-Opetition. Currency Doubleday, New York, 1996.

[9] D. L. Brito and P. R. Hartley. Consumer rationality and credit cards. The Journal of Political Economy, 103(2):400-433, April 1995.

[10] H. Cai. A theory of joint asset ownership. The RAND Journal of Economics, 34(1):63-77, Spring 2003.

[11] S. Chakravorti. Theory of credit card networks: A survey of the literature. Review of Network Economics, 2:50-68, June 2003.

[12] C. J. Corbett and U. S. Karmarkar. Competition and structure in serial supply chains with deterministic demand. Management Science, 47(7):966-978, July 2001.

[13] R. P. DeGennaro. Merchant acquirers and payment card processors: A look inside the black box. Economic Review, 91(1):27-42, The First Quarter 2006. 
[14] T. S. H. Driessen. Cooperative Games, Solutions and Applications. Kluwer Academic Publishers, Boston, 1988.

[15] J. Ericsson and O. Renault. Liquidity and credit risk. The Journal of Finance, 61(5):2219-2250, 2006.

[16] D. B. Gillies. Solutions to general non-zero-sum games. In A. W. Tucker and R. D. Luce, editors, Contributions to the Theory of Games, pages 47-85. Princeton University Press, Princeton, New Jersey, 1959.

[17] S. H. Gow and L. C. Thomas. Interchange fees for bank ATM networks. Naval Research Logistics, 45:407-417, 1998.

[18] D. Hartman. Advantages of the credit card payment systems, 2010. Retrieved June 12, 2012, from http://www. ehow.com/list_6062811_advantages-credit-card-payment-systems.html.

[19] House of Representatives of the U.S.A. Congress takes on credit card interchange fees, 2009. http://www.hr2695.com/ (URL last accessed on June 12, 2012).

[20] R. M. Hunt. An introduction to the economics of payment card networks. Review of Network Economics, 2(2):80-96, June 2003.

[21] M. Leng and M. Parlar. Allocation of cost savings in a three-level supply chain with demand information sharing: A cooperative-game approach. Operations Research, 57(1):200-213, JanuaryFebruary 2009.

[22] M. Leng and M. Parlar. Analytic solution for the nucleolus of a three-player cooperative game. Naval Research Logistics, 57:667-672, October 2010.

[23] A. P. Lerner. The concept of monopoly and the measurement of monopoly power. The Review of Economic Studies, 1(3):157-175, June 1934.

[24] N. Lim and T. Ho. Designing price contracts for boundedly rational customers: Does the number of blocks matter? Marketing Science, 26(3):312-326, May-June 2007.

[25] R. J. Mann. Credit cards and debit cards in the United States and Japan. Monetary and Economic Studies, 20(1):123-160, January 2002.

[26] M. Maschler, B. Peleg, and L. Shapley. Geometric properties of the kernel, nucleolus, and related solution concepts. Mathematics of Operations Research, 4(4):303-338, November 1979.

[27] MasterCard.com. Interchange rates \& fees, 2011. http://www.mastercard.com/us/merchant/ support/interchange_rates.html (URL last accessed on June 12, 2012).

[28] N. Megiddo. On the nonmonotonicity of the bargaining set, the kernel and the nucleolus of a game. SIAM Journal on Applied Mathematics, 27(2):355-358, September 1974.

[29] J. Nash. The bargaining problem. Econometrica, 18:155-162, 1950. 
[30] L. Petrosjan and G. Zaccour. Time-consistent Shapley value allocation of pollution cost reduction. Journal of Economic Dynamics \& Control, 27:381-398, 2003.

[31] R. L. Phillips. Pricing and Revenue Optimization. Stanford Business Press, Stanford, 2005.

[32] R. A. Prager, M. D. Manuszak, E. K. Kiser, and R. Borzekowski. Interchange fees and payment card networks: Economics, industry developments, and policy issues, 2009. www. federalreserve. gov/pubs/feds/2009/200923/200923pap.pdf (URL last accessed on June 12, 2012).

[33] J. Rochet and J. Tirole. Cooperation among competitors: Some economics of payment card associations. The RAND Journal of Economics, 33(4):549-570, Winter 2002.

[34] R. Schmalensee. Payment systems and interchange fees. The Journal of Industrial Economics, 50(2):103-122, June 2002.

[35] D. Schmeidler. The nucleolus of a characteristic function game. SIAM Journal on Applied Mathematics, 17:1163-1170, 1969.

[36] R. J. Sexton. The formation of cooperatives: A game-theoretic approach with implications for cooperative finance, decision making, and stability. American Journal of Agricultural Economics, 68(2):214-225, 1986.

[37] L. S. Shapley. A value for n-person games. In H. W. Kuhn and A. W. Tucker, editors, Contributions to the Theory of Games II, pages 307-317. Princeton University Press, Princeton, 1953.

[38] L. S. Shapley. Cores of convex games. International Journal of Game Theory, 1(1):11-26, December 1971.

[39] J. Small and J. Wright. The bilateral negotiation of interchange fees in payment schemes. Working paper, NECG and University of Auckland, 2002.

[40] P. D. Straffin. Game Theory and Strategy. The Mathematical Association of America, Washington, D.C., 1993.

[41] G. Sun. The Division of Labor in Economics: A History. Routledge, New York, 2012.

[42] L. C. Thomas. Dividing credit-card costs fairly. IMA Journal of Mathematics Applied in Business E Industry, 4:19-33, 1992.

[43] D. M. Topkis. Supermodularity and Complementarity. Princeton University Press, New Jersey, 1998.

[44] M. Verdier. Interchange fees in payment card systems: A survey of the literature. To appear in Journal of Economic Surveys, 2010.

[45] J. von Neumann and O. Morgenstern. Theory of Games and Economic Behaviour. Princeton University Press, Princeton, 1944. 
[46] J. Wang. The Theory of Games. Oxford University Press, New York, 1988.

[47] B. Woolsey and M. Schulz. Credit card statistics, industry facts, debt statistics, July 2011. http://www.creditcards.com/credit-card-news/ credit-card-industry-facts-personal-debt-statistics-1276.php\#Fees (URL last accessed on June 12, 2012).

[48] J. Wright. The determinants of optimal interchange fees in payment systems. The Journal of Industrial Economics, LII:0022-1821, March 2004.

[49] H. P. Young. Monotonic solutions of cooperative games. International Journal of Game Theory, 14(2):65-72, June 1985.

[50] T. J. Zywicki. The economics of payment card interchange fees and the limits of regulation. Technical report, International Center for Law \& Economics, Portland, U.S.A., June 2010. ICLE Financial Regulatory Program White Paper Series. 